\title{
Non-local halo bias with and without massive neutrinos
}

\author{
Matteo Biagetti ${ }^{(1)}$, Vincent Desjacques ${ }^{(1)}$, Alex Kehagias ${ }^{(1,2)}$ and Antonio Riotto ${ }^{(1)}$ \\ (1) Université de Genève, Department of Theoretical Physics and Center for Astroparticle Physics (CAP), \\ 24 quai E. Ansermet, CH-1211 Geneva 4, Switzerland and \\ (2) Physics Division, National Technical University of Athens, 15780 Zografou Campus, Athens, Greece
}

(Dated: October 4, 2018)

\begin{abstract}
Understanding the biasing between the clustering properties of halos and the underlying dark matter distribution is important for extracting cosmological information from ongoing and upcoming galaxy surveys. While on sufficiently larges scales the halo overdensity is a local function of the mass density fluctuations, on smaller scales the gravitational evolution generates non-local terms in the halo density field. We characterize the magnitude of these contributions at third-order in perturbation theory by identifying the coefficients of the non-local invariant operators, and extend our calculation to include non-local (Lagrangian) terms induced by a peak constraint. We apply our results to describe the scale-dependence of halo bias in cosmologies with massive neutrinos. The inclusion of gravity-induced non-local terms and, especially, a Lagrangian $k^{2}$-contribution is essential to reproduce the numerical data accurately. We use the peak-background split to derive the numerical values of the various bias coefficients from the excursion set peak mass function. For neutrino masses in the range $0 \leq \sum_{i} m_{\nu_{i}} \leq 0.6 \mathrm{eV}$, we are able to fit the data with a precision of a few percents up to $k=0.3 \mathrm{~h} \mathrm{Mpc}^{-1}$ without any free parameter.
\end{abstract}

\section{INTRODUCTION}

Cosmological parameter estimation from galaxy clustering data is hampered by galaxy biasing, i.e. by the fact that galaxies do not perfectly trace the underlying mass distribution. Various theoretical arguments and outcomes of numerical simulations both suggest that, on sufficiently large scales, the galaxy overdensity $\delta_{\mathrm{h}}(\vec{x}, \tau)$ can be written as a generic function $f[\delta(\vec{x}, \tau)]$ of the mass density perturbation $\delta(\vec{x}, \tau)$. This function can be Taylor-expanded, with the unknown coefficients in the series defining the so-called bias parameters

$$
\delta_{\mathrm{h}}(\vec{x}, \tau)=f[\delta(\vec{x}, \tau)]=b_{1}(\tau) \delta(\vec{x}, \tau)+\frac{b_{2}(\tau)}{2}\left(\delta^{2}(\vec{x}, \tau)-\left\langle\delta^{2}(\vec{x}, \tau)\right\rangle\right)+\cdots .
$$

We use the subscript (h), which stands for halos, because galaxies form within Dark Matter (DM) halos and, therefore, understanding the clustering properties of the halos is a key step towards an accurate description of galaxy biasing. Furthermore, this is simpler problem since DM halos collapse under the action of gravity solely.

The local model [1] described in, e.g., (I.1) is however incomplete: there is indeed no a priori reason why the halo density contrast should be only a local function of the matter density contrast. Indeed, already at second-order in perturbation theory, the gravitational evolution generates a term quadratic in the tidal tensor and, therefore, nonlocal in the density field. This term is absent in the initial conditions. This point was made in Refs. [2 5 , for the matter density contrast and subsequently investigated in the context of halo bias in Refs. 6 6 13. In Refs. [8, 13, in particular, it was pointed out that the symmetries (essentially extended Galilean and Lifshitz symmetries) present in the dynamical equations for the halo and DM systems allow to construct a set of invariant operators which should appear in the halo bias expansion, precisely because they are allowed by the symmetries of the problem. These invariants lead to non-local bias contributions, which numerical simulations have already detected at the quadratic level and found to agree well with the prediction of perturbation theory [11, 12. In this paper we compute the non-local bias coefficients in the basis of invariant non-local bias operators at third-order in perturbation theory by using the Lagrangian Bias parameters generated within the peak-background split model.

Although the magnitude of the non-local bias terms is small relative to the linear halo bias, upcoming large scale structure data will be sensitive to them. In particular, the impact of the non-local bias, if not accounted for, could mimic the $k$-dependent suppression of the growth rate in cosmologies with massive neutrinos [14 19] (see [20, 21] for detailed reviews of the subject). Their influence on the spatial distribution of DM halos has been recently scrutinized in a series of papers 22 24] using large N-body simulations that incorporate massive neutrinos as an extra set of particles (see also the recent work of [25]). Massive neutrinos generate a scale-dependent bias in the power spectrum of DM halos. As we will see, this effect is somewhat degenerate with the signature left by the various non-local bias terms, which must be taken into account in order to reproduce the N-body data with good accuracy (i.e. at the $\leq 5 \%$ level).

The paper is organized as follows. In Sec. II] we present our computation of the non-local bias at third-order in perturbation theory as well as the calculation of the Lagrangian bias parameters through the peak-background split 
model. In Sec. III we discuss the halo bias in the presence of massive neutrinos and argue that contributions from nonlocal Lagrangian and gravity bias must be accounted for in order to fit the numerical data. We conclude in Sec. IV.

\section{NON-LOCAL BIAS UP TO THIRD-ORDER}

In this section we extend the analysis of [8, 11, 12] and compute the non-local bias coefficients up to third order in perturbation theory. Our starting point is the evolution over cosmic time and in Eulerian space of the halo progenitors - the so-called proto-halos - until their virialization. The basic idea is that, while their shapes and topology change as a function of time (smaller substructures gradually merge to form the final halo), their centre of mass moves along a well-defined trajectory determined by the surrounding mass density field [26. Therefore, unlike virialized halos that undergo merging, by construction proto-halos always preserve their identity. Their total number is therefore conserved over time, such that we can write a continuity equation for their number density

$$
\dot{\delta}_{\mathrm{h}}(\vec{x}, \tau)+\vec{\nabla} \cdot\left[\left(1+\delta_{\mathrm{h}}(\vec{x}, \tau)\right) \vec{v}(\vec{x}, \tau)\right]=0
$$

We may subtract from it the DM mass conservation equation of motion

$$
\dot{\delta}(\vec{x}, \tau)+\vec{\nabla} \cdot[(1+\delta(\vec{x}, \tau)) \vec{v}(\vec{x}, \tau)]=0
$$

where we have assumed unbiased halo velocity (we will relax this assumption later), to get

$$
\dot{\delta}_{\mathrm{h}}(\vec{x}, \tau)-\dot{\delta}(\vec{x}, \tau)+\vec{\nabla} \cdot\left[\left(\delta_{\mathrm{h}}(\vec{x}, \tau)-\delta(\vec{x}, \tau)\right) \vec{v}(\vec{x}, \tau)\right]=0
$$

This is the fundamental equation which we will solve order by order in perturbation theory, and which gives rise to a non-local bias expansion. For the sake of simplicity, we will restrict ourselves to a matter-dominated Universe and denote by $\vec{x}$ the comoving spatial coordinates, $\tau=\int \mathrm{d} t / a$ the conformal time where $a$ the scale factor in the FRW metric, and $\mathcal{H}=\mathrm{d} \ln a / \mathrm{d} \tau$ the conformal expansion rate. In addition, $\delta(\vec{x}, \tau)=(\rho(\vec{x}, \tau) / \bar{\rho}-1)$ is the overdensity over the mean matter density $\bar{\rho}, \delta_{\mathrm{h}}(\vec{x}, \tau)$ stands for the halo counterpart, and $\vec{v}(\vec{x}, \tau)$ is the common peculiar velocity. In

the following we will also denote by $\Phi(\vec{x}, \tau)$ the gravitational potential induced by density fluctuations. We begin with a review of the first- and second-order calculations before deriving the third-order nonlocal biases.

\section{A. First-order}

At first-order from Eq. II.3 we get

$$
\dot{\delta}_{\mathrm{h}}^{(1)}(\vec{x}, \tau)-\dot{\delta}^{(1)}(\vec{x}, \tau)=0
$$

or

$$
\delta_{\mathrm{h}}^{(1)}(\vec{x}, \tau)=\delta_{\mathrm{h}}^{(1)}\left(\vec{x}, \tau_{i}\right)+\delta^{(1)}(\vec{x}, \tau)-\delta^{(1)}\left(\vec{x}, \tau_{i}\right)
$$

where $\tau_{i}$ is some initial time. We assume that the initial bias expansion is local and depends only on the linear DM density contrast through the (Lagrangian) bias coefficients

$$
\begin{aligned}
\delta_{\mathrm{h}}\left(\vec{x}, \tau_{i}\right)= & \sum_{\ell} \frac{b_{\ell}^{\mathrm{L}}\left(\tau_{i}\right)}{\ell !}\left(\delta^{(1)}\left(\vec{x}, \tau_{i}\right)\right)^{\ell} \\
= & \sum_{\ell} \frac{b_{\ell}^{\mathrm{L}}(\tau)}{\ell !}\left(\delta^{(1)}(\vec{x}, \tau)\right)^{\ell} \\
\simeq & b_{1}^{\mathrm{L}}(\tau) \delta^{(1)}(\vec{x}, \tau)+\frac{1}{2} b_{2}^{\mathrm{L}}(\tau)\left(\delta^{(1)}(\vec{x}, \tau)\right)^{2}+ \\
& \frac{1}{3 !} b_{3}^{\mathrm{L}}(\tau)\left(\delta^{(1)}(\vec{x}, \tau)\right)^{3}+\cdots,
\end{aligned}
$$


where $b_{\ell}^{\mathrm{L}}(\tau)=b_{\ell}^{\mathrm{L}}\left(\tau_{i}\right)\left(a\left(\tau_{i}\right) / a(\tau)\right)^{\ell}$. Using $\delta_{\mathrm{h}}^{(1)}\left(\vec{x}, \tau_{i}\right)=b_{1}^{\mathrm{L}}(\tau) \delta(\vec{x}, \tau)$, we obtain the standard result

$$
\delta_{\mathrm{h}}^{(1)}(\vec{x}, \tau) \simeq\left(1+b_{1}^{\mathrm{L}}(\tau)\right) \delta^{(1)}(\vec{x}, \tau)
$$

\section{B. Second-order}

At second-order we may use the first-order result to write Eq. (II.3) in the form

$$
\begin{aligned}
\dot{\delta}_{\mathrm{h}}^{(2)}(\vec{x}, \tau) & -\dot{\delta}^{(2)}(\vec{x}, \tau) \\
& +\vec{\nabla} \cdot\left[\left(\delta_{\mathrm{h}}^{(1)}(\vec{x}, \tau)-\delta^{(1)}(\vec{x}, \tau)\right) \vec{v}^{(1)}(\vec{x}, \tau)\right]=0,
\end{aligned}
$$

which is solved by

$$
\begin{aligned}
\delta_{\mathrm{h}}^{(2)}(\vec{x}, \tau) & =\delta_{\mathrm{h}}^{(2)}\left(\vec{x}, \tau_{i}\right)+\delta^{(2)}(\vec{x}, \tau)-\int^{\tau} \mathrm{d} \eta b_{1}^{\mathrm{L}}(\eta) \vec{\nabla} \cdot\left[\delta^{(1)}(\vec{x}, \eta) \vec{v}^{(1)}(\vec{x}, \eta)\right] \\
& \simeq \frac{1}{2} b_{2}^{\mathrm{L}}(\tau)\left(\delta^{(1)}(\vec{x}, \tau)\right)^{2}+\delta^{(2)}(\vec{x}, \tau)-\int^{\tau} \mathrm{d} \eta b_{1}^{\mathrm{L}}(\eta) \vec{\nabla} \cdot\left[\delta^{(1)}(\vec{x}, \eta) \vec{v}^{(1)}(\vec{x}, \eta)\right] \\
& =\frac{1}{2} b_{2}^{\mathrm{L}}(\tau)\left(\delta^{(1)}(\vec{x}, \tau)\right)^{2}+\delta^{(2)}(\vec{x}, \tau)-\int^{\tau} \mathrm{d} \eta b_{1}^{\mathrm{L}}(\eta) \vec{\nabla} \delta^{(1)}(\vec{x}, \eta) \cdot \vec{v}^{(1)}(\vec{x}, \eta)+\int^{\tau} \mathrm{d} \eta b_{1}^{\mathrm{L}}(\eta) \delta^{(1)}(\vec{x}, \eta) \dot{\delta}^{(1)}(\vec{x}, \eta) \\
& =\frac{1}{2} b_{2}^{\mathrm{L}}(\tau)\left(\delta^{(1)}(\vec{x}, \tau)\right)^{2}+\delta^{(2)}(\vec{x}, \tau)-\frac{\tau}{2} b_{1}^{\mathrm{L}}(\tau) \vec{\nabla} \delta^{(1)}(\vec{x}, \tau) \cdot \vec{v}^{(1)}(\vec{x}, \tau)+b_{1}^{\mathrm{L}}(\tau)\left(\delta^{(1)}(\vec{x}, \tau)\right)^{2}
\end{aligned}
$$

or

$$
\begin{aligned}
\delta_{\mathrm{h}}^{(2)}(\vec{x}, \tau) & =-\frac{1}{\mathcal{H}} b_{1}^{\mathrm{L}}(\tau) \vec{\nabla} \delta^{(1)}(\vec{x}, \tau) \cdot \vec{v}^{(1)}(\vec{x}, \tau) \\
& +\frac{1}{2} b_{2}^{\mathrm{L}}(\tau)\left(\delta^{(1)}(\vec{x}, \tau)\right)^{2}+\delta^{(2)}(\vec{x}, \tau)+b_{1}^{\mathrm{L}}(\tau)\left(\delta^{(1)}(\vec{x}, \tau)\right)^{2}
\end{aligned}
$$

To perform the time integrals, we have used the scalings provided by the first-order quantities (in matter-domination)

$$
\begin{aligned}
v_{i}^{(1)}(\vec{x}, \tau) & =-\frac{\tau}{3} \partial_{i} \varphi(\vec{x})=-\frac{2}{3 \mathcal{H}} \partial_{i} \varphi(\vec{x}), \\
\delta^{(1)}(\vec{x}, \tau) & =\frac{\tau^{2}}{6} \nabla^{2} \varphi(\vec{x})=\frac{2}{3 \mathcal{H}^{2}} \nabla^{2} \varphi(\vec{x}),
\end{aligned}
$$

where $\varphi(\vec{x})$ is the initial condition for the gravitational potential $\Phi(\vec{x}, \tau)$. In order to elaborate further the second-order halo density contrast, we remind the reader that [27, 28.

$$
\begin{aligned}
\delta^{(2)}(\vec{x}, \tau) & =\frac{\tau^{4}}{2 \cdot 126}\left[5\left(\nabla^{2} \varphi(\vec{x})\right)^{2}+2 \partial_{k} \partial_{p} \varphi(\vec{x}) \partial^{k} \partial^{p} \varphi(\vec{x})+7 \partial^{i} \varphi(\vec{x}) \nabla^{2} \partial_{i} \varphi(\vec{x})\right] \\
& =\frac{5}{7}\left(\delta^{(1)}(\vec{x}, \tau)\right)^{2}+\frac{8}{63 \mathcal{H}^{4}} \partial_{k} \partial_{p} \varphi(\vec{x}) \partial^{k} \partial^{p} \varphi(\vec{x})-\frac{1}{\mathcal{H}} \vec{\nabla} \delta^{(1)}(\vec{x}, \tau) \cdot \vec{v}(1)(\vec{x}, \tau)
\end{aligned}
$$

and define the non-local bias operator

$$
s_{i j}(\vec{x}, \tau)=\frac{2}{3 \mathcal{H}^{2}} \partial_{i} \partial_{j} \Phi(\vec{x}, \tau)-\frac{1}{3} \delta_{i j} \delta(\vec{x}, \tau)
$$

to get 


$$
\partial_{k} \partial_{p} \varphi(\vec{x}) \partial^{k} \partial^{p} \varphi(\vec{x})=\frac{9 \mathcal{H}^{4}}{4}\left(s^{(1)}(\vec{x}, \tau)\right)^{2}+\frac{3 \mathcal{H}^{4}}{4}\left(\delta^{(1)}(\vec{x}, \tau)\right)^{2} .
$$

From this expression we deduce

$$
\delta^{(2)}(\vec{x}, \tau)=\frac{17}{21}\left(\delta^{(1)}(\vec{x}, \tau)\right)^{2}+\frac{2}{7}\left(s^{(1)}(\vec{x}, \tau)\right)^{2}-\frac{1}{\mathcal{H}} \vec{\nabla} \delta^{(1)}(\vec{x}, \tau) \cdot \vec{v}^{(1)}(\vec{x}, \tau) .
$$

We finally arrive at

$$
\delta_{\mathrm{h}}^{(2)}(\vec{x}, \tau) \simeq\left(1+b_{1}^{\mathrm{L}}(\tau)\right) \delta^{(2)}(\vec{x}, \tau)+\left(\frac{1}{2} b_{2}^{\mathrm{L}}(\tau)+\frac{4}{21} b_{1}^{\mathrm{L}}(\tau)\right)\left(\delta^{(1)}(\vec{x}, \tau)\right)^{2}-\frac{2}{7} b_{1}^{\mathrm{L}}(\tau)\left(s^{(1)}(\vec{x}, \tau)\right)^{2} .
$$

This result reproduces exactly the one derived in Refs. [6, 12] and shows that at second-order in perturbation theory the halo overdensity is not a local function of the underlying matter overdensity.

\section{Third-order}

We now proceed to the original part of the computation at third-order. The equation to solve is

$$
\dot{\delta}_{\mathrm{h}}^{(3)}(\vec{x}, \tau)-\dot{\delta}^{(3)}(\vec{x}, \tau)+\vec{\nabla} \cdot\left[\left(\delta_{\mathrm{h}}^{(1)}(\vec{x}, \tau)-\delta^{(1)}(\vec{x}, \tau)\right) \vec{v}^{(2)}(\vec{x}, \tau)\right]+\vec{\nabla} \cdot\left[\left(\delta_{\mathrm{h}}^{(2)}(\vec{x}, \tau)-\delta^{(2)}(\vec{x}, \tau)\right) \vec{v}^{(1)}(\vec{x}, \tau)\right]=0
$$

where 28 ]

$$
\begin{aligned}
\vec{v}_{i}^{(2)}(\vec{x}, \tau) & =\frac{\tau^{3}}{18}\left[-\partial_{i} \partial_{j} \varphi(\vec{x}) \partial^{j} \varphi(\vec{x})-\frac{3}{7} \frac{\partial_{i}}{\nabla^{2}}\left(\left(\nabla^{2} \varphi(\vec{x})\right)^{2}-\partial_{k} \partial_{p} \varphi(\vec{x}) \partial^{k} \partial^{p} \varphi(\vec{x})\right)\right] \\
\vec{\nabla} \cdot \vec{v}_{i}^{(2)}(\vec{x}, \tau) & =\frac{\tau^{3}}{9}\left[-\partial_{j} \nabla^{2} \varphi(\vec{x}) \partial^{j} \varphi(\vec{x})-\partial_{i} \partial_{j} \varphi(\vec{x}) \partial^{i} \partial^{j} \varphi(\vec{x})-\frac{3}{7}\left(\left(\nabla^{2} \varphi(\vec{x})\right)^{2}-\partial_{k} \partial_{p} \varphi(\vec{x}) \partial^{k} \partial^{p} \varphi(\vec{x})\right)\right] \\
& =\frac{\tau^{3}}{18}\left[-\partial_{j} \nabla^{2} \varphi(\vec{x}) \partial^{j} \varphi(\vec{x})-\frac{4}{7} \partial_{i} \partial_{j} \varphi(\vec{x}) \partial^{i} \partial^{j} \varphi(\vec{x})-\frac{3}{7}\left(\nabla^{2} \varphi(\vec{x})\right)^{2}\right] .
\end{aligned}
$$

Eq. (II.17) gives

$$
\begin{aligned}
\delta_{\mathrm{h}}^{(3)}(\vec{x}, \tau) & =\delta_{\mathrm{h}}^{(3)}\left(\vec{x}, \tau_{i}\right)+\delta^{(3)}(\vec{x}, \tau) \\
& -\int^{\tau} \mathrm{d} \eta b_{1}^{\mathrm{L}}(\eta) \vec{\nabla} \cdot\left[\delta^{(1)}(\vec{x}, \eta) \vec{v}^{(2)}(\vec{x}, \eta)\right] \\
& -\int^{\tau} \mathrm{d} \eta b_{1}^{\mathrm{L}}(\eta) \vec{\nabla} \cdot\left[\delta^{(2)}(\vec{x}, \eta) \vec{v}^{(1)}(\vec{x}, \eta)\right] \\
& -\int^{\tau} \mathrm{d} \eta \vec{\nabla} \cdot\left[\left(\left(\frac{1}{2} b_{2}^{\mathrm{L}}(\eta)+\frac{4}{21} b_{1}^{\mathrm{L}}(\eta)\right)\left(\delta^{(1)}(\vec{x}, \eta)\right)^{2}-\frac{2}{7} b_{1}^{\mathrm{L}}(\tau)\left(s^{(1)}(\vec{x}, \tau)\right)^{2}\right) \vec{v}^{(1)}(\vec{x}, \eta)\right],
\end{aligned}
$$

or

$$
\begin{aligned}
\delta_{\mathrm{h}}^{(3)}(\vec{x}, \tau) & =\frac{1}{3 !} b_{3}^{\mathrm{L}}(\tau)\left(\delta^{(1)}(\vec{x}, \tau)\right)^{3}+\delta^{(3)}(\vec{x}, \tau) \\
& -\int^{\tau} \mathrm{d} \eta b_{1}^{\mathrm{L}}(\eta) \vec{\nabla} \delta^{(1)}(\vec{x}, \eta) \cdot \vec{v}^{(2)}(\vec{x}, \eta)
\end{aligned}
$$




$$
\begin{aligned}
& -\int^{\tau} \mathrm{d} \eta b_{1}^{\mathrm{L}}(\eta) \delta^{(1)}(\vec{x}, \eta) \vec{\nabla} \cdot \vec{v}^{(2)}(\vec{x}, \eta) \\
& -\int^{\tau} \mathrm{d} \eta b_{1}^{\mathrm{L}}(\eta) \vec{\nabla} \delta^{(2)}(\vec{x}, \eta) \cdot \vec{v}^{(1)}(\vec{x}, \eta) \\
& -\int^{\tau} \mathrm{d} \eta b_{1}^{\mathrm{L}}(\eta) \delta^{(2)}(\vec{x}, \eta) \vec{\nabla} \cdot \vec{v}^{(1)}(\vec{x}, \eta) \\
& -\int^{\tau} \mathrm{d} \eta\left(\frac{1}{2} b_{2}^{\mathrm{L}}(\eta)+\frac{4}{21} b_{1}^{\mathrm{L}}(\eta)\right) \vec{\nabla}\left[\left(\delta^{(1)}(\vec{x}, \eta)\right)^{2}\right] \cdot \vec{v}^{(1)}(\vec{x}, \eta) \\
& -\int^{\tau} \mathrm{d} \eta\left(\frac{1}{2} b_{2}^{\mathrm{L}}(\eta)+\frac{4}{21} b_{1}^{\mathrm{L}}(\eta)\right)\left(\delta^{(1)}(\vec{x}, \eta)\right)^{2} \vec{\nabla} \cdot \vec{v}^{(1)}(\vec{x}, \eta) \\
& +\frac{2}{7} \int^{\tau} \mathrm{d} \eta b_{1}^{\mathrm{L}}(\eta) \vec{\nabla}\left(s^{(1)}(\vec{x}, \eta)\right)^{2} \cdot \vec{v}^{(1)}(\vec{x}, \eta) \\
& +\frac{2}{7} \int^{\tau} \mathrm{d} \eta b_{1}^{\mathrm{L}}(\eta)\left(s^{(1)}(\vec{x}, \eta)\right)^{2} \vec{\nabla} \cdot \vec{v}^{(1)}(\vec{x}, \eta) .
\end{aligned}
$$

We can integrate over time to obtain

$$
\begin{aligned}
\delta_{\mathrm{h}}^{(3)}(\vec{x}, \tau) & =\frac{1}{3 !} b_{3}^{\mathrm{L}}(\tau)\left(\delta^{(1)}(\vec{x}, \tau)\right)^{3}+\delta^{(3)}(\vec{x}, \tau) \\
& -\frac{\tau}{4} b_{1}^{\mathrm{L}}(\tau) \vec{\nabla} \delta^{(1)}(\vec{x}, \tau) \cdot \vec{v}^{(2)}(\vec{x}, \tau) \\
& -\frac{\tau}{4} b_{1}^{\mathrm{L}}(\tau) \delta^{(1)}(\vec{x}, \tau) \vec{\nabla} \cdot \vec{v}^{(2)}(\vec{x}, \tau) \\
& -\frac{\tau}{4} b_{1}^{\mathrm{L}}(\tau) \vec{\nabla} \delta^{(2)}(\vec{x}, \tau) \cdot \vec{v}^{(1)}(\vec{x}, \tau) \\
& -\frac{\tau}{4} b_{1}^{\mathrm{L}}(\tau) \delta^{(2)}(\vec{x}, \tau) \vec{\nabla} \cdot \vec{v}^{(1)}(\vec{x}, \tau) \\
& -\left(\frac{\tau}{4} b_{2}^{\mathrm{L}}(\tau)+\frac{\tau}{21} b_{1}^{\mathrm{L}}(\tau)\right)\left\{\vec{\nabla}\left[\left(\delta^{(1)}(\vec{x}, \tau)\right)^{2}\right] \cdot \vec{v}^{(1)}(\vec{x}, \tau)+\left(\delta^{(1)}(\vec{x}, \tau)\right)^{2} \vec{\nabla} \cdot \vec{v}^{(1)}(\vec{x}, \tau)\right\} \\
& +\frac{\tau}{14} b_{1}^{\mathrm{L}}(\tau) \vec{\nabla}\left(s^{(1)}(\vec{x}, \tau)\right)^{2} \cdot \vec{v}^{(1)}(\vec{x}, \tau) \\
& +\frac{\tau}{14} b_{1}^{\mathrm{L}}(\tau)\left(s^{(1)}(\vec{x}, \tau)\right)^{2} \vec{\nabla} \cdot \vec{v}^{(1)}(\vec{x}, \tau) .
\end{aligned}
$$

Now, the mass conservation equation for the DM at third-order reads

$$
\begin{aligned}
\dot{\delta}^{(3)}(\vec{x}, \tau)+\vec{\nabla} \cdot \vec{v}^{(3)}(\vec{x}, \tau) & =-\vec{\nabla} \delta^{(2)}(\vec{x}, \tau) \cdot \vec{v}^{(1)}(\vec{x}, \tau)-\vec{\nabla} \delta^{(1)}(\vec{x}, \tau) \cdot \vec{v}^{(2)}(\vec{x}, \tau) \\
& -\delta^{(2)}(\vec{x}, \tau) \vec{\nabla} \cdot \vec{v}^{(1)}(\vec{x}, \tau)-\delta^{(1)}(\vec{x}, \tau) \vec{\nabla} \cdot \vec{v}^{(2)}(\vec{x}, \tau) .
\end{aligned}
$$

Since $\delta^{(3)}(\vec{x}, \tau)$ scales like $a^{3}$, we can rewrite it as

$$
\begin{aligned}
3 \mathcal{H} \delta^{(3)}(\vec{x}, \tau)+\vec{\nabla} \cdot \vec{v}^{(3)}(\vec{x}, \tau) & =-\left[\vec{\nabla} \delta^{(2)}(\vec{x}, \tau) \cdot \vec{v}^{(1)}(\vec{x}, \tau)+\vec{\nabla} \delta^{(1)}(\vec{x}, \tau) \cdot \vec{v}^{(2)}(\vec{x}, \tau)\right. \\
& \left.+\delta^{(2)}(\vec{x}, \tau) \vec{\nabla} \cdot \vec{v}^{(1)}(\vec{x}, \tau)+\delta^{(1)}(\vec{x}, \tau) \vec{\nabla} \cdot \vec{v}^{(2)}(\vec{x}, \tau)\right]
\end{aligned}
$$

Eq. (II.21 then becomes

$$
\begin{aligned}
\delta_{\mathrm{h}}^{(3)}(\vec{x}, \tau) & =\frac{1}{3 !} b_{3}^{\mathrm{L}}(\tau)\left(\delta^{(1)}(\vec{x}, \tau)\right)^{3}+\left(\frac{3}{2} b_{1}^{\mathrm{L}}(\tau)+1\right) \delta^{(3)}(\vec{x}, \tau)+\frac{1}{2 \mathcal{H}} b_{1}^{\mathrm{L}}(\tau) \theta^{(3)}(\vec{x}, \tau) \\
& -\left(\frac{\tau}{4} b_{2}^{\mathrm{L}}(\tau)+\frac{\tau}{21} b_{1}^{\mathrm{L}}(\tau)\right)\left\{\vec{\nabla}\left[\left(\delta^{(1)}(\vec{x}, \tau)\right)^{2}\right] \cdot \vec{v}^{(1)}(\vec{x}, \tau)+\left(\delta^{(1)}(\vec{x}, \tau)\right)^{2} \vec{\nabla} \cdot \vec{v}^{(1)}(\vec{x}, \tau)\right\}
\end{aligned}
$$




$$
\begin{aligned}
& +\frac{\tau}{14} b_{1}^{\mathrm{L}}(\tau) \vec{\nabla}\left(s^{(1)}(\vec{x}, \tau)\right)^{2} \cdot \vec{v}^{(1)}(\vec{x}, \tau) \\
& +\frac{\tau}{14} b_{1}^{\mathrm{L}}(\tau)\left(s^{(1)}(\vec{x}, \tau)\right)^{2} \vec{\nabla} \cdot \vec{v}^{(1)}(\vec{x}, \tau),
\end{aligned}
$$

where $\theta(\vec{x}, \tau)=\vec{\nabla} \cdot \vec{v}(\vec{x}, \tau)$ satisfies at any order in perturbation theory the DM momentum equation

$$
\dot{\theta}(\vec{x}, \tau)+\mathcal{H} \theta(\vec{x}, \tau)+\partial^{j} v_{i}(\vec{x}, \tau) \partial^{i} v_{j}(\vec{x}, \tau)+\vec{v}(\vec{x}, \tau) \cdot \vec{\nabla} \theta(\vec{x}, \tau)=-\frac{3}{2} \mathcal{H}^{2} \delta(\vec{x}, \tau) .
$$

Following Refs. [8, 13, we introduce another non-local coefficient

$$
t_{i j}(\vec{x}, \tau)=-\frac{1}{\mathcal{H}}\left(\partial_{i} v_{j}(\vec{x}, \tau)-\frac{1}{3} \delta_{i j} \theta(\vec{x}, \tau)\right)-s_{i j}(\vec{x}, \tau)
$$

It is traceless and vanishes at first-order in perturbation theory as

$$
s_{i j}^{(1)}(\vec{x}, \tau)=-\frac{1}{\mathcal{H}} \partial_{i} v_{j}^{(1)}(\vec{x}, \tau)-\frac{1}{3} \delta_{i j} \delta^{(1)}(\vec{x}, \tau),
$$

and therefore

$$
t_{i j}^{(1)}(\vec{x}, \tau)=\frac{1}{3} \delta_{i j}\left(\frac{1}{\mathcal{H}} \theta^{(1)}(\vec{x}, \tau)+\delta^{(1)}(\vec{x}, \tau)\right)=0 .
$$

This implies that $t^{2}(\vec{x}, \tau)=t_{i j}(\vec{x}, \tau) t^{i j}(\vec{x}, \tau)$ is fourth-order and we can neglect it here and henceforth. In particular, up to third-order,

$$
\partial^{j} v_{i}(\vec{x}, \tau) \partial^{i} v_{j}(\vec{x}, \tau)=-2 \mathcal{H}^{2} t(\vec{x}, \tau) \cdot s(\vec{x}, \tau)+\frac{1}{3} \theta^{2}(\vec{x}, \tau)+\mathcal{H}^{2} s^{2}(\vec{x}, \tau)
$$

The equation for $\theta(\vec{x}, \tau)$ then becomes

$$
\dot{\theta}(\vec{x}, \tau)+\mathcal{H} \theta(\vec{x}, \tau)-2 \mathcal{H}^{2} t(\vec{x}, \tau) \cdot s(\vec{x}, \tau)+\frac{1}{3} \theta^{2}(\vec{x}, \tau)+\mathcal{H}^{2} s^{2}(\vec{x}, \tau)+\vec{v}(\vec{x}, \tau) \cdot \vec{\nabla} \theta(\vec{x}, \tau)=-\frac{3}{2} \mathcal{H}^{2} \delta(\vec{x}, \tau)
$$

Since

$$
\begin{aligned}
\vec{\nabla} \cdot \vec{v}^{(2)}(\vec{x}, \tau) & =\frac{\tau^{3}}{18}\left[-\partial_{j} \nabla^{2} \varphi(\vec{x}) \partial^{j} \varphi(\vec{x})-\frac{4}{7} \partial_{i} \partial_{j} \varphi(\vec{x}) \partial^{i} \partial^{j} \varphi(\vec{x})-\frac{3}{7}\left(\nabla^{2} \varphi(\vec{x})\right)^{2}\right] \\
& =\vec{\nabla} \delta^{(1)}(\vec{x}, \tau) \cdot \vec{v}^{(1)}(\vec{x}, \tau)-\frac{4}{7} \mathcal{H}\left(s^{(1)}(\vec{x}, \tau)\right)^{2}-\frac{13}{21} \mathcal{H}\left(\delta^{(1)}(\vec{x}, \tau)\right)^{2},
\end{aligned}
$$

we have

$$
-\frac{1}{\mathcal{H}} \vec{\nabla} \cdot \vec{v}^{(2)}(\vec{x}, \tau)-\delta^{(2)}(\vec{x}, \tau)=\frac{2}{7}\left(s^{(1)}(\vec{x}, \tau)\right)^{2}-\frac{4}{21}\left(\delta^{(1)}(\vec{x}, \tau)\right)^{2}
$$

and it is convenient to define two new non-local bias operators [8]

$$
\eta(\vec{x}, \tau)=-\frac{\theta(\vec{x}, \tau)}{\mathcal{H}}-\delta(\vec{x}, \tau)
$$

and

$$
\psi(\vec{x}, \tau)=\eta(\vec{x}, \tau)-\frac{2}{7} s^{2}(\vec{x}, \tau)+\frac{4}{21} \delta^{2}(\vec{x}, \tau)
$$


By construction $\eta(\vec{x}, \tau)$ is a second-order quantity and $\psi(\vec{x}, \tau)$ is third-order quantity. Eq. II.24 becomes then

$$
\begin{aligned}
\delta_{\mathrm{h}}^{(3)}(\vec{x}, \tau) & =\frac{1}{3 !} b_{3}^{\mathrm{L}}(\tau)\left(\delta^{(1)}(\vec{x}, \tau)\right)^{3}+\left(\frac{3}{2} b_{1}^{\mathrm{L}}(\tau)+1\right) \delta^{(3)}(\vec{x}, \tau) \\
& +\frac{1}{2} b_{1}^{\mathrm{L}}(\tau)\left(-\psi^{(3)}(\vec{x}, \tau)-\delta^{(3)}(\vec{x}, \tau)-\frac{4}{7} s^{(1)}(\vec{x}, \tau) s^{(2)}(\vec{x}, \tau)+\frac{8}{21} \delta^{(1)}(\vec{x}, \tau) \delta^{(2)}(\vec{x}, \tau)\right) \\
& -\frac{1}{\mathcal{H}}\left(\frac{1}{2} b_{2}^{\mathrm{L}}(\tau)+\frac{2}{21} b_{1}^{\mathrm{L}}(\tau)\right)\left\{\vec{\nabla}\left[\left(\delta^{(1)}(\vec{x}, \tau)\right)^{2}\right] \cdot \vec{v}^{(1)}(\vec{x}, \tau)+\left(\delta^{(1)}(\vec{x}, \tau)\right)^{2} \vec{\nabla} \cdot \vec{v}^{(1)}(\vec{x}, \tau)\right\} \\
& +\frac{1}{7 \mathcal{H}} b_{1}^{\mathrm{L}}(\tau) \vec{\nabla}\left(s^{(1)}(\vec{x}, \tau)\right)^{2} \cdot \vec{v}^{(1)}(\vec{x}, \tau) \\
& +\frac{1}{7 \mathcal{H}} b_{1}^{\mathrm{L}}(\tau)\left(s^{(1)}(\vec{x}, \tau)\right)^{2} \vec{\nabla} \cdot \vec{v}^{(1)}(\vec{x}, \tau) .
\end{aligned}
$$

Using Eq. II.15 we can rewrite it as

$$
\begin{aligned}
\delta_{\mathrm{h}}^{(3)}(\vec{x}, \tau) & =\frac{1}{3 !} b_{3}^{\mathrm{L}}(\tau)\left(\delta^{(1)}(\vec{x}, \tau)\right)^{3}+\left(1+b_{1}^{\mathrm{L}}(\tau)\right) \delta^{(3)}(\vec{x}, \tau) \\
& +\frac{1}{2} b_{1}^{\mathrm{L}}(\tau)\left(-\psi^{(3)}(\vec{x}, \tau)-\frac{4}{7} s^{(1)}(\vec{x}, \tau) \cdot s^{(2)}(\vec{x}, \tau)+\frac{8}{21} \delta^{(1)}(\vec{x}, \tau) \delta^{(2)}(\vec{x}, \tau)\right) \\
& -\frac{1}{\mathcal{H}}\left(\frac{1}{2} b_{2}^{\mathrm{L}}(\tau)+\frac{2}{21} b_{1}^{\mathrm{L}}(\tau)\right) \delta^{(1)}(\vec{x}, \tau)\left\{2 \vec{\nabla} \delta^{(1)}(\vec{x}, \tau) \cdot \vec{v}^{(1)}(\vec{x}, \tau)-\mathcal{H}\left(\delta^{(1)}(\vec{x}, \tau)\right)^{2}\right\} \\
& +\frac{1}{7 \mathcal{H}} b_{1}^{\mathrm{L}}(\tau) \vec{\nabla}\left(s^{(1)}(\vec{x}, \tau)\right)^{2} \cdot \vec{v}^{(1)}(\vec{x}, \tau) \\
& +\frac{1}{7 \mathcal{H}} b_{1}^{\mathrm{L}}(\tau)\left(s^{(1)}(\vec{x}, \tau)\right)^{2} \vec{\nabla} \cdot \vec{v}^{(1)}(\vec{x}, \tau),
\end{aligned}
$$

or

$$
\begin{aligned}
\delta_{\mathrm{h}}^{(3)}(\vec{x}, \tau) & =\left(1+b_{1}^{\mathrm{L}}(\tau)\right) \delta^{(3)}(\vec{x}, \tau) \\
& +2\left(\frac{1}{2} b_{2}^{\mathrm{L}}(\tau)+\frac{4}{21} b_{1}^{\mathrm{L}}(\tau)\right) \delta^{(1)}(\vec{x}, \tau) \delta^{(2)}(\vec{x}, \tau) \\
& +\left[\frac{1}{3 !} b_{3}^{\mathrm{L}}(\tau)-\frac{13}{21}\left(\frac{1}{2} b_{2}^{\mathrm{L}}(\tau)+\frac{2}{21} b_{1}^{\mathrm{L}}(\tau)\right)\right]\left(\delta^{(1)}(\vec{x}, \tau)\right)^{3} \\
& +\frac{1}{2} b_{1}^{\mathrm{L}}(\tau)\left(-\psi^{(3)}(\vec{x}, \tau)-\frac{4}{7} s^{(1)}(\vec{x}, \tau) \cdot s^{(2)}(\vec{x}, \tau)\right) \\
& -\frac{4}{7}\left(\frac{1}{2} b_{2}^{\mathrm{L}}(\tau)+\frac{29}{84} b_{1}^{\mathrm{L}}(\tau)\right) \delta^{(1)}(\vec{x}, \tau)\left(s^{(1)}(\vec{x}, \tau)\right)^{2} \\
& +\frac{1}{7 \mathcal{H}} b_{1}^{\mathrm{L}}(\tau) \vec{\nabla}\left(s^{(1)}(\vec{x}, \tau)\right)^{2} \cdot \vec{v}^{(1)}(\vec{x}, \tau) .
\end{aligned}
$$

Let us concentrate on the last term of the expression (II.37). We can operate a series of manipulations on it

$$
\begin{aligned}
\vec{\nabla}\left(s^{(1)}(\vec{x}, \tau)\right)^{2} \cdot \vec{v}^{(1)}(\vec{x}, \tau)= & 2 s_{i j}^{(1)}(\vec{x}, \tau) v_{k}^{(1)}(\vec{x}, \tau) \partial^{k} s_{i j}^{(1)}(\vec{x}, \tau) \\
= & -\frac{2}{\mathcal{H}} s_{i j}^{(1)}(\vec{x}, \tau) v_{k}^{(1)}(\vec{x}, \tau) \partial^{k} \partial_{i} v_{j}^{(1)}(\vec{x}, \tau) \\
= & -\frac{2}{\mathcal{H}} s_{i j}^{(1)}(\vec{x}, \tau)\left[\partial_{i}\left(v_{k}^{(1)}(\vec{x}, \tau) \partial^{k} v_{j}^{(1)}(\vec{x}, \tau)\right)-\partial_{i} v_{k}^{(1)}(\vec{x}, \tau) \partial^{k} v_{j}^{(1)}(\vec{x}, \tau)\right] \\
= & -\frac{2}{\mathcal{H}} s_{i j}^{(1)}(\vec{x}, \tau)\left[-\partial_{i} \partial_{j} \Phi^{(2)}(\vec{x}, \tau)-\partial_{i} \dot{v}_{j}^{(2)}(\vec{x}, \tau)-\mathcal{H} \partial_{i} v_{j}^{(2)}(\vec{x}, \tau)\right] \\
& +2 \mathcal{H} s_{i j}^{(1)}(\vec{x}, \tau)\left(s_{i k}^{(1)}(\vec{x}, \tau)+\frac{1}{3} \delta_{i k} \delta^{(1)}(\vec{x}, \tau)\right)\left(s_{k j}^{(1)}(\vec{x}, \tau)+\frac{1}{3} \delta_{k j} \delta^{(1)}(\vec{x}, \tau)\right) \\
= & -\frac{2}{\mathcal{H}} s_{i j}^{(1)}(\vec{x}, \tau)\left(-\frac{3 \mathcal{H}^{2}}{2} s_{i j}^{(2)}(\vec{x}, \tau)\right)-\frac{2}{\mathcal{H}} s_{i j}^{(1)}(\vec{x}, \tau)\left(-\frac{5}{2} \mathcal{H} \partial_{i} v_{j}^{(2)}(\vec{x}, \tau)\right)
\end{aligned}
$$




$$
\begin{aligned}
& +2 \mathcal{H} s_{i j}^{(1)}(\vec{x}, \tau)\left(s_{i k}^{(1)}(\vec{x}, \tau) s_{k j}^{(1)}(\vec{x}, \tau)+\frac{2}{3} s_{i j}^{(1)}(\vec{x}, \tau) \delta^{(1)}(\vec{x}, \tau)\right) \\
= & 3 \mathcal{H} s_{i j}^{(1)}(\vec{x}, \tau) s_{i j}^{(2)}(\vec{x}, \tau)+5 s_{i j}^{(1)}(\vec{x}, \tau) \mathcal{H}\left(\frac{1}{3 \mathcal{H}} \delta_{i j} \theta^{(2)}(\vec{x}, \tau)-t_{i j}^{(2)}(\vec{x}, \tau)-s_{i j}^{(2)}(\vec{x}, \tau)\right) \\
& +2 \mathcal{H}\left(s^{(1)}(\vec{x}, \tau)\right)^{3}+\frac{4}{3} \mathcal{H}\left(s^{(1)}(\vec{x}, \tau)\right)^{2} \delta^{(1)}(\vec{x}, \tau) \\
= & -2 \mathcal{H} s_{i j}^{(1)}(\vec{x}, \tau) s_{i j}^{(2)}(\vec{x}, \tau)-5 \mathcal{H} s_{i j}^{(1)}(\vec{x}, \tau) t_{i j}^{(2)}(\vec{x}, \tau) \\
+ & 2 \mathcal{H}\left(s^{(1)}(\vec{x}, \tau)\right)^{3}+\frac{4}{3} \mathcal{H}\left(s^{(1)}(\vec{x}, \tau)\right)^{2} \delta^{(1)}(\vec{x}, \tau) .
\end{aligned}
$$

Therefore

$$
\begin{aligned}
\frac{1}{7 \mathcal{H}} b_{1}^{\mathrm{L}}(\tau) \vec{\nabla}\left(s^{(1)}(\vec{x}, \tau)\right)^{2} \cdot \vec{v}^{(1)}(\vec{x}, \tau)= & -\frac{2}{7} b_{1}^{\mathrm{L}}(\tau) s_{i j}^{(1)}(\vec{x}, \tau) s_{i j}^{(2)}(\vec{x}, \tau)-\frac{5}{7} b_{1}^{\mathrm{L}}(\tau) s_{i j}^{(1)}(\vec{x}, \tau) t_{i j}^{(2)}(\vec{x}, \tau) \\
& +\frac{2}{7} b_{1}^{\mathrm{L}}(\tau)\left(s^{(1)}(\vec{x}, \tau)\right)^{3}+\frac{4}{21} b_{1}^{\mathrm{L}}(\tau)\left(s^{(1)}(\vec{x}, \tau)\right)^{2} \delta^{(1)}(\vec{x}, \tau),
\end{aligned}
$$

so that

$$
\begin{aligned}
\delta_{\mathrm{h}}^{(3)}(\vec{x}, \tau) & =\left(1+b_{1}^{\mathrm{L}}(\tau)\right) \delta^{(3)}(\vec{x}, \tau) \\
& +2\left(\frac{1}{2} b_{2}^{\mathrm{L}}(\tau)+\frac{4}{21} b_{1}^{\mathrm{L}}(\tau)\right) \delta^{(1)}(\vec{x}, \tau) \delta^{(2)}(\vec{x}, \tau) \\
& +\left[\frac{1}{3 !} b_{3}^{\mathrm{L}}(\tau)-\frac{13}{21}\left(\frac{1}{2} b_{2}^{\mathrm{L}}(\tau)+\frac{2}{21} b_{1}^{\mathrm{L}}(\tau)\right)\right]\left(\delta^{(1)}(\vec{x}, \tau)\right)^{3} \\
& +\frac{1}{2} b_{1}^{\mathrm{L}}(\tau)\left(-\psi^{(3)}(\vec{x}, \tau)-\frac{8}{7} s^{(1)}(\vec{x}, \tau) \cdot s^{(2)}(\vec{x}, \tau)\right) \\
& -\frac{4}{7}\left(\frac{1}{2} b_{2}^{\mathrm{L}}(\tau)+\frac{2}{81} b_{1}^{\mathrm{L}}(\tau)\right) \delta^{(1)}(\vec{x}, \tau)\left(s^{(1)}(\vec{x}, \tau)\right)^{2} \\
& -\frac{5}{7} b_{1}^{\mathrm{L}}(\tau) s_{i j}^{(1)}(\vec{x}, \tau) t_{i j}^{(2)}(\vec{x}, \tau)+\frac{2}{7} b_{1}^{\mathrm{L}}(\tau)\left(s^{(1)}(\vec{x}, \tau)\right)^{3} .
\end{aligned}
$$

Combining the results (II.7), (II.16), and (II.40), we finally get

$$
\begin{aligned}
\delta_{\mathrm{h}}(\vec{x}, \tau) & =\left(1+b_{1}^{\mathrm{L}}(\tau)\right) \delta(\vec{x}, \tau)+\left(\frac{1}{2} b_{2}^{\mathrm{L}}(\tau)+\frac{4}{21} b_{1}^{\mathrm{L}}(\tau)\right) \delta^{2}(\vec{x}, \tau)-\frac{2}{7} b_{1}^{\mathrm{L}}(\tau) s^{2}(\vec{x}, \tau) \\
& +\left[\frac{1}{3 !} b_{3}^{\mathrm{L}}(\tau)-\frac{13}{21}\left(\frac{1}{2} b_{2}^{\mathrm{L}}(\tau)+\frac{2}{21} b_{1}^{\mathrm{L}}(\tau)\right)\right] \delta^{3}(\vec{x}, \tau) \\
& -\frac{1}{2} b_{1}^{\mathrm{L}}(\tau) \psi-\frac{4}{7}\left(\frac{1}{2} b_{2}^{\mathrm{L}}(\tau)+\frac{2}{81} b_{1}^{\mathrm{L}}(\tau)\right) \delta(\vec{x}, \tau) s^{2}(\vec{x}, \tau) \\
& -\frac{5}{7} b_{1}^{\mathrm{L}}(\tau) s(\vec{x}, \tau) \cdot t(\vec{x}, \tau)+\frac{2}{7} b_{1}^{\mathrm{L}}(\tau) s^{3}(\vec{x}, \tau) .
\end{aligned}
$$

The halo density contrast can thus be written as

$$
\delta_{\mathrm{h}}(\vec{x}, \tau)=b_{1} \delta(\vec{x}, \tau)+\frac{1}{2 !} b_{2} \delta^{2}(\vec{x}, \tau)+\frac{1}{3 !} b_{3} \delta^{3}(\vec{x}, \tau)+\frac{1}{2} b_{s^{2}} s^{2}(\vec{x}, \tau)+b_{\psi} \psi(\vec{x}, \tau)+b_{s t} s(\vec{x}, \tau) \cdot t(\vec{x}, \tau)+\cdots,
$$

where the relevant bias coefficients are

$$
b_{1}=1+b_{1}^{\mathrm{L}}, b_{2}=b_{2}^{\mathrm{L}}+\frac{8}{21} b_{1}^{\mathrm{L}}, b_{s^{2}}=-\frac{4}{7} b_{1}^{\mathrm{L}}, b_{\psi}=-\frac{1}{2} b_{1}^{\mathrm{L}}, b_{s t}=-\frac{5}{7} b_{1}^{\mathrm{L}} .
$$

This is the main result of this Section. 


\section{Scale-dependent bias}

So far, we have not considered the possibility that the Lagrangian bias factors may be scale-dependent. In peak theory for instance, the peak constraint induces $k$-dependent corrections at all orders in the bias coefficients 9 , 2931. Moreover, peak velocities are statistically biased at linear order, and this bias propagates to higher order owing to gravity mode-coupling [9, 32. Even though calculations within the peak formalism are relatively tedious, the modifications brought by the peak constraint can actually be easily implemented in any Lagrangian approach $[9$, 30]: the scale-independent Lagrangian bias factors $b_{n}^{\mathrm{L}}$ should be replaced (in Fourier space) by the scale-dependent functions $c_{n}^{\mathrm{L}}\left(\vec{k}_{1}, \ldots \vec{k}_{n}, \tau_{i}\right)$. At the lowest orders,

$$
\begin{aligned}
c_{1}^{\mathrm{L}}\left(k, \tau_{i}\right)= & b_{10}^{\mathrm{L}}\left(\tau_{i}\right)+b_{01}^{\mathrm{L}}\left(\tau_{i}\right) k^{2}, \\
c_{2}^{\mathrm{L}}\left(\vec{k}_{1}, \vec{k}_{2}, \tau_{i}\right)=\{ & b_{20}^{\mathrm{L}}\left(\tau_{i}\right)+b_{11}^{\mathrm{L}}\left(\tau_{i}\right)\left(k_{1}^{2}+k_{2}^{2}\right)+b_{02}^{\mathrm{L}}\left(\tau_{i}\right) k_{1}^{2} k_{2}^{2} \\
& \left.\quad-2 \chi_{10}^{\mathrm{L}}\left(\tau_{i}\right)\left(\vec{k}_{1} \cdot \vec{k}_{2}\right)+\chi_{01}^{\mathrm{L}}\left(\tau_{i}\right)\left[3\left(\vec{k}_{1} \cdot \vec{k}_{2}\right)^{2}-k_{1}^{2} k_{2}^{2}\right]\right\} .
\end{aligned}
$$

As a result, the local Lagrangian bias expansion generalizes to

$$
\begin{aligned}
\delta_{\mathrm{h}}\left(\vec{x}, \tau_{i}\right) & =b_{10}^{\mathrm{L}}\left(\tau_{i}\right) \delta\left(\vec{x}, \tau_{i}\right)-b_{01}^{\mathrm{L}}\left(\tau_{i}\right) \vec{\nabla}^{2} \delta\left(\vec{x}, \tau_{i}\right)+\frac{1}{2} b_{20}^{\mathrm{L}}\left(\tau_{i}\right) \delta^{2}\left(\vec{x}, \tau_{i}\right) \\
& -b_{11}^{\mathrm{L}}\left(\tau_{i}\right) \delta\left(\vec{x}, \tau_{i}\right) \vec{\nabla}^{2} \delta\left(\vec{x}, \tau_{i}\right)+\frac{1}{2} b_{02}^{\mathrm{L}}\left(\tau_{i}\right)\left[\vec{\nabla}^{2} \delta\left(\vec{x}, \tau_{i}\right)\right]^{2} \\
& +\chi_{10}^{\mathrm{L}}\left(\tau_{i}\right)(\vec{\nabla} \delta)^{2}\left(\vec{x}, \tau_{i}\right)+\frac{1}{2} \chi_{01}^{\mathrm{L}}\left(\tau_{i}\right)\left[3 \partial_{i} \partial_{j} \delta-\delta_{i j} \vec{\nabla}^{2} \delta\right]^{2}\left(\vec{x}, \tau_{i}\right)+\ldots,
\end{aligned}
$$

where the various bias factors $b_{i j}^{\mathrm{L}}$ and $\chi_{i j}^{\mathrm{L}}$ can be obtained from a peak-background split applied to the halo mass function (see 30] for details).

In addition, the Zel'dovich gravity mode-coupling kernels $F_{n}^{\mathrm{ZA}}\left(\vec{k}_{1}, \ldots, \vec{k}_{n}\right)$ should be replaced by

$$
\mathcal{F}_{n}^{\mathrm{ZA}}\left(\vec{k}_{1}, \ldots, \vec{k}_{n}\right)=F_{n}^{\mathrm{ZA}}\left(\vec{k}_{1}, \ldots, \vec{k}_{n}\right) \times b_{v}\left(k_{1}\right) \ldots b_{v}\left(k_{n}\right) .
$$

Here, $b_{v}(k)$ is the linear velocity bias. In the peak model for instance, it is $b_{v}(k)=1-R_{v}^{2} k^{2}$ where

$$
R_{v}^{2}=\frac{\sigma_{0}^{2}}{\sigma_{1}^{2}}
$$

is a characteristic scale that is proportional to the Lagrangian radius of a halo, and $\sigma_{j}^{2} \equiv\left\langle k^{2 j}\right\rangle$ are moments of the matter power spectrum (smoothed on the Lagrangian halo scale). The $k^{2}$-dependence arises from requiring invariance under rotations. In fact, the linear peak velocities can be thought of as arising from the continuous, local relation [9, 29]

$$
\vec{v}_{\mathrm{h}}\left(\vec{x}, \tau_{i}\right)=\vec{v}\left(\vec{x}, \tau_{i}\right)-R_{v}^{2} \vec{\nabla} \delta\left(\vec{x}, \tau_{i}\right) .
$$

This development could be generalized to include all the higher order terms consistent with the symmetry of the problem. However, since we are mainly interested in the first-order scale-dependent corrections that dominate at relatively small $k$, we will postpone such a study to future work. Eq. (II.47) reflects the fact that, in the peak approach, the linear velocity bias remains constant throughout time 9]. By contrast, the fluid approximation to halos and dark matter predicts that any initial velocity bias should decay rapidly to unity [11. Given that this discrepancy has not been resolved yet (see, however, Ref. 33), we will assume that the velocity bias remains constant in order to write down expressions more general than those obtained within the fluid approximation.

At the first order, Eq.(II.7) generalizes easily to

$$
\delta_{\mathrm{h}}^{(1)}(\vec{x}, \tau) \simeq\left(1+b_{10}^{\mathrm{L}}(\tau)\right) \delta^{(1)}(\vec{x}, \tau)+\left(R_{v}^{2}-b_{01}^{\mathrm{L}}(\tau)\right) \vec{\nabla}^{2} \delta^{(1)}(\vec{x}, \tau),
$$


which follows from the linearized continuity equation $\delta^{(1)} \propto-\vec{\nabla} \cdot \vec{v}^{(1)}$. This result reproduces the findings of 9 , who explicitly took into account the peak constraint. Note also that $b_{01}^{\mathrm{L}}(\tau)=b_{01}^{\mathrm{L}}\left(\tau_{i}\right)\left(a\left(\tau_{i}\right) / a(\tau)\right)$. As can be seen, the amplitude of the contribution proportional to $k^{2}$ scales as $R_{v}^{2}-b_{01}^{\mathrm{L}}(\tau)$, which is generally non-zero. Therefore, we shall expect this $k^{2}$-dependence to appear at sufficiently small scales in the halo bias.

At the second order, the halo overabundance $\delta_{\mathrm{h}}(\vec{x}, \tau)$ with scale-dependent spatial and velocity bias can be computed by combining the results of Ref. [9] (derived in the Zel'dovich approximation) with those of [10] (derived at higher order in Lagrangian PT). The second-order halo overdensity takes the form

$$
\begin{aligned}
\delta_{\mathrm{h}}(\vec{x}, \tau)=\frac{1}{2} \int \frac{d^{3} q_{1}}{(2 \pi)^{3}} \int \frac{d^{3} q_{2}}{(2 \pi)^{3}}\left\{\mathcal{F}_{2}^{\mathrm{ZA}}\left(\vec{q}_{1}, \vec{q}_{2}\right)+\frac{a\left(\tau_{i}\right)}{a(\tau)}\left[\mathcal{F}_{1}^{\mathrm{ZA}}\left(\vec{q}_{1}\right) c_{1}\left(q_{2}, \tau_{i}\right)+\mathcal{F}_{1}^{\mathrm{ZA}}\left(\vec{q}_{2}\right) c_{1}\left(q_{1}, \tau_{i}\right)\right]\right. \\
\left.+\frac{a^{2}\left(\tau_{i}\right)}{a^{2}(\tau)} c_{2}\left(\vec{q}_{1}, \vec{q}_{2}, \tau_{i}\right)+\frac{2}{7}-\frac{3}{7}\left(\mu^{2}-\frac{1}{3}\right)\right\} \delta^{(1)}\left(\vec{q}_{1}, \tau\right) \delta^{(1)}\left(\vec{q}_{2}, \tau\right) \delta_{\mathrm{D}}\left(\vec{k}-\vec{q}_{1}-\vec{q}_{2}\right),
\end{aligned}
$$

where we have momentarily set $\delta^{(1)} \equiv \nabla^{2} \phi^{(1)}$ so that it resembles the well-known PT expression. The various contributions can be reduced to a combination of local and nonlocal quantities analogously to the calculation performed above. For instance, $(1 / 2)\left(a\left(\tau_{i}\right) / a(\tau)\right)\left[\mathcal{F}_{1}^{\mathrm{ZA}}\left(\vec{q}_{1}\right) c_{1}\left(q_{2}, \tau_{i}\right)+1 \leftrightarrow 2\right]$ includes a term proportional to $(1 / 2) b_{01}^{\mathrm{L}}(\tau)\left[q_{2}^{2} \frac{\vec{k} \cdot \hat{\mathbf{q}}_{1}}{q_{1}}+1 \leftrightarrow 2\right]$ which, after some manipulations, becomes

$$
\begin{aligned}
b_{01}^{\mathrm{L}}(\tau) \vec{\nabla}\left(\nabla^{2} \delta^{(1)} \vec{\nabla}^{-1} \delta^{(1)}\right)=- & b_{01}^{\mathrm{L}}(\tau) \nabla^{2} \delta^{(2)}(\vec{k}, \tau)+\frac{9}{7} b_{01}^{\mathrm{L}}(\tau) \delta^{(1)} \nabla^{2} \delta^{(1)}+\frac{18}{7} b_{01}^{\mathrm{L}}(\tau)\left(\vec{\nabla} \delta^{(1)}\right)^{2} \\
& +\frac{6}{7} b_{01}^{\mathrm{L}}(\tau)\left(\frac{2}{3 \mathcal{H}^{2}} \partial_{i} \partial_{j} \Phi-\frac{1}{3} \delta_{i j} \delta\right)\left(\partial_{i} \partial_{j} \delta-\frac{1}{3} \delta_{i j} \nabla^{2} \delta\right) \\
& +\frac{4}{7} b_{01}^{\mathrm{L}}(\tau)\left(\frac{2}{3 \mathcal{H}^{2}} \partial_{i} \partial_{j} \partial_{k} \Phi-\frac{1}{3} \delta_{i j} \partial_{k} \delta\right)^{2} .
\end{aligned}
$$

We have restored the factors of $3 /\left(2 \mathcal{H}^{2}\right)$ to be consistent with the units employed throughout the paper. The first term in the right-hand side adds to $-b_{01}^{\mathrm{L}} \nabla^{2} \delta^{(1)}$ in Eq. III.50) to yield $-b_{01}^{\mathrm{L}} \nabla^{2} \delta$, where $\delta$ is the mass density fluctuations at second order. The following two terms contribute to the Eulerian biases $b_{11}$ and $b_{02}$. The last two terms are new nonlocal bias contributions, which are however heavily suppressed relative to $s^{2}(\vec{x}, \tau)$ as they carry additional powers of $\vec{k}$. The remaining terms in the right-hand side of Eq. II.51) can be written analogously. For the purpose of the present work however, Eq. (II.50) and its $k$-dependent contribution at linear order are sufficient. Therefore, we generalize Eq. (II.42) to

$$
\begin{aligned}
\delta_{\mathrm{h}}(\vec{x}, \tau)=b_{10} \delta & (\vec{x}, \tau)-b_{01} \nabla^{2} \delta(\vec{x}, \tau)+\frac{1}{2 !} b_{20} \delta^{2}(\vec{x}, \tau) \\
& +\frac{1}{2} b_{s^{2}} s^{2}(\vec{x}, \tau)+b_{\psi} \psi(\vec{x}, \tau)+b_{s t} s(\vec{x}, \tau) \cdot t(\vec{x}, \tau)+\cdots
\end{aligned}
$$

where

$$
b_{10}=1+b_{10}^{\mathrm{L}}, \quad b_{01}=-R_{v}^{2}+b_{01}^{\mathrm{L}}, \quad b_{20}=b_{20}^{\mathrm{L}}+\frac{8}{21} b_{10}^{\mathrm{L}}, \quad b_{s^{2}}=-\frac{4}{7} b_{10}^{\mathrm{L}}, \quad b_{\psi}=-\frac{1}{2} b_{10}^{\mathrm{L}}, \quad b_{s t}=-\frac{5}{7} b_{10}^{\mathrm{L}} .
$$

This is the model we will consider hereafter. As we will see shortly, a scale-dependent bias at linear order appears necessary to explain recent numerical measurements of halo bias with massive neutrinos.

\section{HALO BIAS IN THE PRESENCE OF MASSIVE NEUTRINOS}

Refs. 22 24 investigated the impact of massive neutrinos on the spatial distribution of dark matter halos and galaxies using large box-size N-body simulations that incorporate massive neutrinos as an extra set of particles. They found that massive neutrinos generate an additional scale-dependence in the halo power spectrum on midly nonlinear scales, in agreement with previous theoretical predictions [34. In this Section, we will compare the scale-dependence induced by massive neutrinos with that generated by gravitational mode-coupling and Lagrangian halo bias. We will show that the latter is substantially steeper, so that it should be relatively easy to isolate the contribution of massive neutrinos from a measurement of the halo power spectrum. 


\section{A. Perturbative approach}

To model the impact of massive neutrinos on the clustering of dark matter halos, we follow [34 37] and assume that the latter trace the cold Dark Matter (CDM) plus baryons fluctuation field, with a linear growth rate suppressed in a scale-dependent way by the massive neutrinos. This approximation is motivated by the smallness of the neutrino masses we consider. For $\sum m_{\nu}<0.6 \mathrm{eV}$, the neutrino free-streaming scale is sufficiently large that the neutrino perturbations remain in the linear regime up to late time. It has been shown to work well in Refs. 222 24. We thus write the total dark matter perturbation as a weighted sum of cold Dark Matter (we will ignore the effect of baryons in what follows, except for the fact that our CDM transfer function is a weighted sum of the baryons + CDM transfer functions) and neutrino fluctuations,

$$
\delta_{\mathrm{m}}=\left(1-f_{\nu}\right) \delta_{\mathrm{c}}+f_{\nu} \delta_{\nu}
$$

where the neutrino overdensity $\delta_{\nu}$ is in the linear regime, and the neutrino fraction $f_{\nu}$ is

$$
f_{\nu}=\frac{\Omega_{\nu}}{\Omega_{c}+\Omega_{\nu}}
$$

Replacing $\delta(\vec{x}, \tau)$ by $\delta_{c}(\vec{x}, \tau)$ in the right-hand side of Eq.(II.53), the halo-mass cross-power spectrum reads

$$
P_{\mathrm{hm}}(k)=\left(b_{10}+b_{01} k^{2}\right) P_{\mathrm{cm}}^{\mathrm{NL}}(k)+\Delta P_{\mathrm{hm}}(k)+P_{\mathrm{cc}}(k) I_{3}(k)
$$

where

$$
P_{\mathrm{cm}}^{\mathrm{NL}}=\frac{P_{\mathrm{mm}}^{\mathrm{NL}}-f_{\nu} P_{\nu \mathrm{m}}}{1-f_{\nu}}=\frac{P_{\mathrm{mm}}^{\mathrm{NL}}-f_{\nu}\left(1-f_{\nu}\right) P_{\mathrm{c} \nu}-f_{\nu}^{2} P_{\nu \nu}}{1-f_{\nu}},
$$

and

$$
\begin{aligned}
\Delta P_{\mathrm{hm}}(k) & =\left(1-f_{\nu}\right) b_{20} \int \frac{\mathrm{d}^{3} q}{(2 \pi)^{3}} P_{\mathrm{cc}}(q) P_{\mathrm{cc}}(|\vec{k}-\vec{q}|) F_{2}(\vec{q}, \vec{k}-\vec{q}) \\
& +\left(1-f_{\nu}\right) b_{s^{2}} \int \frac{\mathrm{d}^{3} q}{(2 \pi)^{3}} P_{\mathrm{cc}}(q) P_{\mathrm{cc}}(|\vec{k}-\vec{q}|) F_{2}(\vec{q}, \vec{k}-\vec{q}) S(\vec{q}, \vec{k}-\vec{q})
\end{aligned}
$$

Here, $P_{\mathrm{cc}}, P_{\nu \nu}$ and $P_{\mathrm{c} \nu}$ are the linear CDM, neutrinos power spectrum and the CDM-neutrinos cross-power spectrum, respectively. We have adopted the notation of Refs. [8, 38] for our definition of

$$
I_{3}(k)=\frac{32}{105}\left(1-f_{\nu}\right)\left(b_{s t}-\frac{5}{2} b_{s^{2}}+\frac{16}{21} b_{\psi}\right) \int \mathrm{d} \ln r \Delta_{\mathrm{cc}}^{2}(k r) I_{R}(r),
$$

being

$$
\begin{aligned}
I_{R}(r) & =I(r)+\frac{5}{6} \\
I(r) & =\frac{105}{32} \int_{-1}^{1} \mathrm{~d} \mu D_{2}(\vec{q}, \vec{k}) S(\vec{q}, \vec{k}-\vec{q})
\end{aligned}
$$

and

$$
\begin{aligned}
F_{2}\left(\vec{q}_{1}, \vec{q}_{2}\right) & =\frac{5}{7}+\frac{1}{2} \frac{\vec{q}_{1} \cdot \vec{q}_{2}}{q_{1} q_{2}}\left(\frac{q_{1}}{q_{2}}+\frac{q_{2}}{q_{1}}\right)+\frac{2}{7}\left(\frac{\vec{q}_{1} \cdot \vec{q}_{2}}{q_{1} q_{2}}\right)^{2}, \\
S\left(\vec{q}_{1}, \vec{q}_{2}\right) & =\left(\frac{\vec{q}_{1} \cdot \vec{q}_{2}}{q_{1} q_{2}}\right)^{2}-\frac{1}{3}, \\
D_{2}\left(\vec{q}_{1}, \vec{q}_{2}\right) & =\frac{2}{7}\left[S\left(\vec{q}_{1}, \vec{q}_{2}\right)-\frac{2}{3}\right] .
\end{aligned}
$$




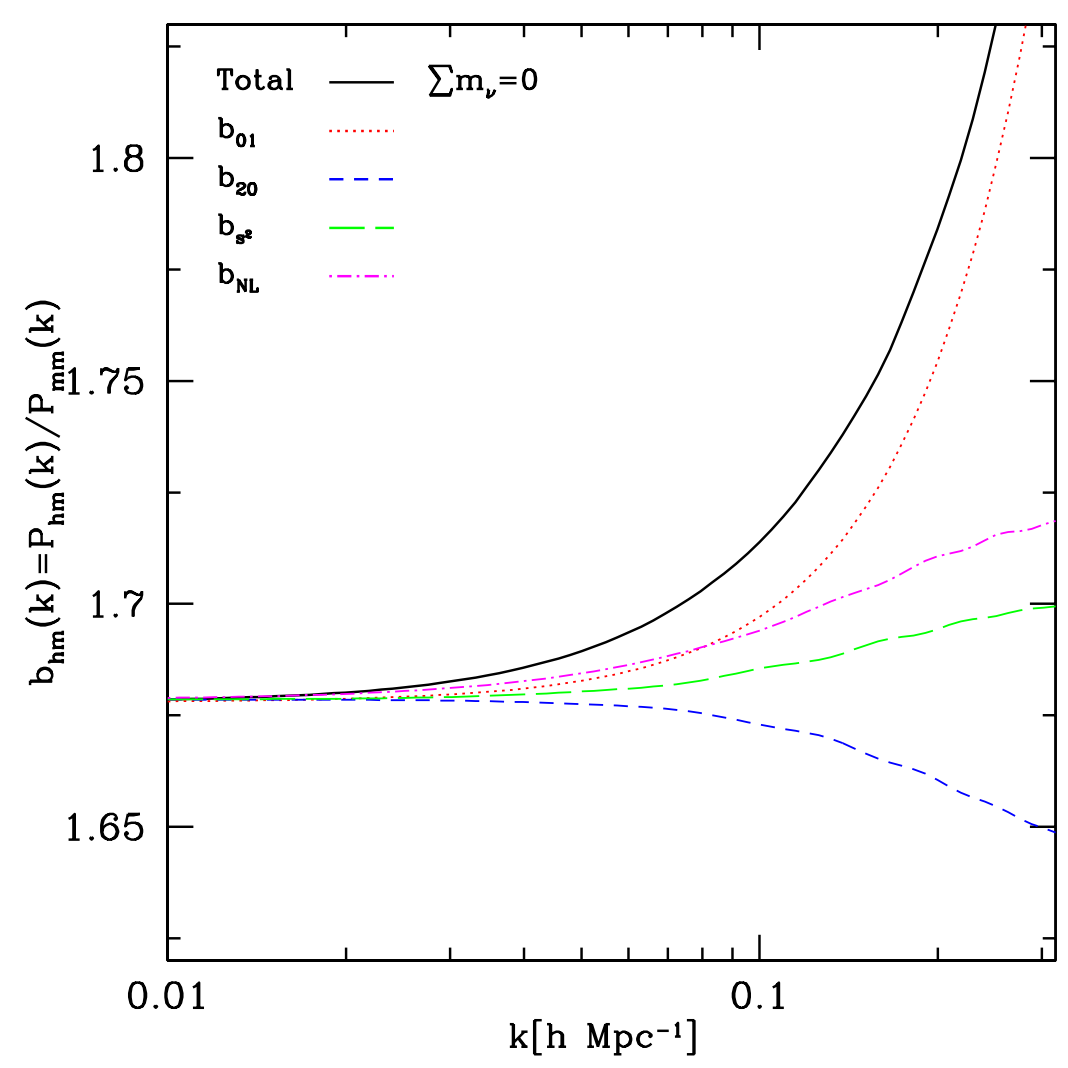

FIG. 1: Halo bias at redshift $z=0$ as a function of wavenumber in the case $\sum m_{\nu}=0$. The different terms that contribute to the scale-dependence bias in Eq.(III.11) are labelled according to the bias parameter they are proportional to (see text). The solid black curve represents the sum of all the contributions, Eq.(III.11). All the bias factors have been computed consistently from the ESP halo mass function and the relations Eq. [II.54).

The latter are the second-order perturbative kernels. Note that, below the neutrino free-streaming scale, the crosspower spectrum $P_{\mathrm{cm}}^{\mathrm{NL}}$ is enhanced by a factor of $\left(1-f_{\nu}\right)^{-1}$ relative to $P_{\mathrm{mm}}^{\mathrm{NL}}$. Following Ref. [8], one should interpret $b_{s t}$ etc. as "renormalized" bias parameters. Eq. (II.43) leads to the following relation

$$
\frac{32}{105}\left(b_{s t}-\frac{5}{2} b_{s^{2}}+\frac{16}{21} b_{\psi}\right)=\frac{32}{315} b_{10}^{\mathrm{L}} \equiv b_{\mathrm{NL}}
$$

Further details regarding the evaluation of these integrals can be found in Appendix 8 ,

The nonlinear mass power spectrum $P_{\mathrm{mm}}^{\mathrm{NL}}$ and the linear $P_{\mathrm{cc}}, P_{\mathrm{c} \nu}$ and $P_{\nu \nu}$, Eqs. (III.3), (III.4) and (III.5), in the presence of massive neutrinos are obtained from the CLASS code [39] (see also Ref. [40] for an overview). We refer the reader to [41] for details about this implementation.

\section{B. Bias Parameters}

To evaluate the halo-matter power spectrum Eq. (III.3), we need predictions for the values of the bias parameters $b_{10}^{\mathrm{L}}, b_{01}^{\mathrm{L}}, b_{20}^{\mathrm{L}}$ together with the scale $R_{v}$ that quantifies the magnitude of the $k$-dependent velocity bias.

For this purpose, we compute all the Lagrangian bias factors using the excursion set peak (ESP) mass function 42 , 43, which has been shown to agree well with simulated halo mass functions constructed with a spherical overdensity (SO) criterion [44 46]. Following [24, 36], we replace the average mass density $\rho_{\mathrm{m}}$ by $\rho_{\text {cdm }}$, and the variance of mass fluctuations $\sigma_{\mathrm{m}}$ by $\sigma_{\mathrm{cc}}$ in the ESP halo mass function. While we refer the reader to the aforementioned references for details, it is worth stressing the following points:

1. The bias parameters depend both on redshift and halo mass. To follow the analysis of [22 24 as closely as possible, we average the Lagrangian bias factors over a suitable range of halo mass, 


$$
b_{i j}^{\mathrm{L}}=\frac{\int_{M_{\min }}^{M_{\max }} b_{i j}^{\mathrm{L}}(M) n_{\mathrm{ESP}}(M) \mathrm{d} M}{\int_{M_{\min }}^{M_{\max }} n_{\mathrm{ESP}}(M) \mathrm{d} M},
$$

where $n_{\mathrm{ESP}}$ is the ESP halo mass function. The mass range is $2 \times 10^{13} h^{-1} M_{\odot}<M<3 \times 10^{15} h^{-1} M_{\odot}$. In the following Table 1 some typical values for the bias parameters for different neutrino masses.

\begin{tabular}{|c|c|c|c|c|}
\hline$\sum_{i} m_{\nu_{i}}(\mathrm{eV})$ & $b_{10}^{\mathrm{L}}$ & $b_{01}^{\mathrm{L}}$ & $R_{v}^{2}$ & $b_{20}^{\mathrm{L}}$ \\
\hline 0 & 0.68 & 12.32 & 10.41 & -0.32 \\
\hline 0.1 & 0.73 & 12.43 & 10.38 & -0.28 \\
\hline 0.2 & 0.80 & 12.55 & 10.33 & -0.22 \\
\hline 0.3 & 0.87 & 12.68 & 10.29 & -0.15 \\
\hline 0.6 & 1.15 & 13.13 & 10.17 & 0.24 \\
\hline
\end{tabular}

TABLE I: Lagrangian bias factors as computed from the ESP mass function for the cosmological models considered here (they are labeled according to the sum of neutrino masses). The bias parameters, defined relative to the linear density field extrapolated at $z=0$, are weighted over the mass range $2 \times 10^{13} h^{-1} M_{\odot}<M<3 \times 10^{15} h^{-1} M_{\odot}$.

2. Here and henceforth, we will use the word "local" when we refer to the simplest bias prescription without any scale dependence. In this case, we set $b_{s^{2}}=b_{\psi}=b_{s t}=b_{01}=0$. Conversely, our scale-dependent bias prescription is summarized by Eq.(II.54) with $R_{v}$ computed from Eq.(II.48). We only retain the nonlocal peak bias $b_{01}^{\mathrm{L}}$ since i) it is the only nonlocal Lagrangian term for which we have the time evolution and ii) several second-order peak bias factors induce $k^{2}$-corrections (see e.g. [9, 10]) that are at least partly degenerate with $\left(b_{01}^{\mathrm{L}}-R_{v}^{2}\right) k^{2}$ over the range of wavenumber considered here.

3. The scale dependencies induced by the peak constraint also propagate to $b_{s^{2}}, b_{\psi}, b_{s t}$ etc. for which the Lagrangian to Eulerian mapping can be derived upon analyzing terms like Eq. (II.52). Since $b_{s^{2}}$ etc. are small however, we do also not expect significant corrections on the scales of interest.

To compare our predictions with the numerical results of $22 \mid 24$, we use a set of $\left(\Omega_{\mathrm{m}}, \Omega_{\nu}\right)$ such that the total mass density $\Omega_{\mathrm{m}}=\Omega_{\mathrm{c}}+\Omega_{\nu}$ is held fixed to 0.2708 while the CDM and neutrino density are varied. The sum of neutrino masses is $\sum m_{\nu}=0,0.1,0.2,0.3$ and $0.6 \mathrm{eV}$, such that $\Omega_{\nu}$ varies between 0 and 0.0131 . We have also adopted $h=0.7$ for the Hubble rate, $n_{s}=1$ for the scalar spectral index, and $A_{s}=2.43 \times 10^{-9}$ for the amplitude of primordial scalar perturbations. The normalization amplitude $\sigma_{8}$ changes in accordance with the sum of neutrino masses [47].

\section{Results}

The quantity we focus on is the halo bias defined as the ratio of the halo-matter cross-power spectrum to the matter auto-power spectrum,

$$
b_{\mathrm{hm}}=\frac{P_{\mathrm{hm}}}{P_{\mathrm{mm}}^{\mathrm{NL}}}=\frac{\left(b_{10}+b_{01} k^{2}\right) P_{\mathrm{cm}}^{\mathrm{NL}}(k)+\Delta P_{\mathrm{hm}}(k)+P_{\mathrm{cc}}(k) I_{3}(k)}{P_{\mathrm{mm}}^{\mathrm{NL}}(k)} .
$$

It is expected to be constant (matching the value of $1+b_{10}^{\mathrm{L}}$ ) on large scales, with a scale dependence arising at smaller scales. The origin of this scale-dependence is twofold: the non-locality discussed in Sec. II and the suppression of the linear growth rate induced by the presence of massive neutrinos. Therefore, we begin by exploring the contributions generated by the non-locality arising from gravity and the peak constraint. Fig. 1 displays the various scale-dependent contributions to the halo bias Eq. [III.11] when the neutrinos are massless, i.e. $\sum m_{\nu}=0$. We have labelled the curves according to the bias parameters that weight each scale-dependent contribution. Namely, $b_{20}$ and $b_{s^{2}}$ denote the two terms of Eq. (III.5) while $b_{\mathrm{NL}}$, defined in Eq. (III.9), indicate the term proportional to $I_{3}(k)$ in Eq. (III.6). Note that $P_{\mathrm{cm}}^{\mathrm{NL}}(k)=P_{\mathrm{mm}}^{\mathrm{NL}}(k)$ in this case. Clearly, the dominant contribution arises from the peak constraint through the 

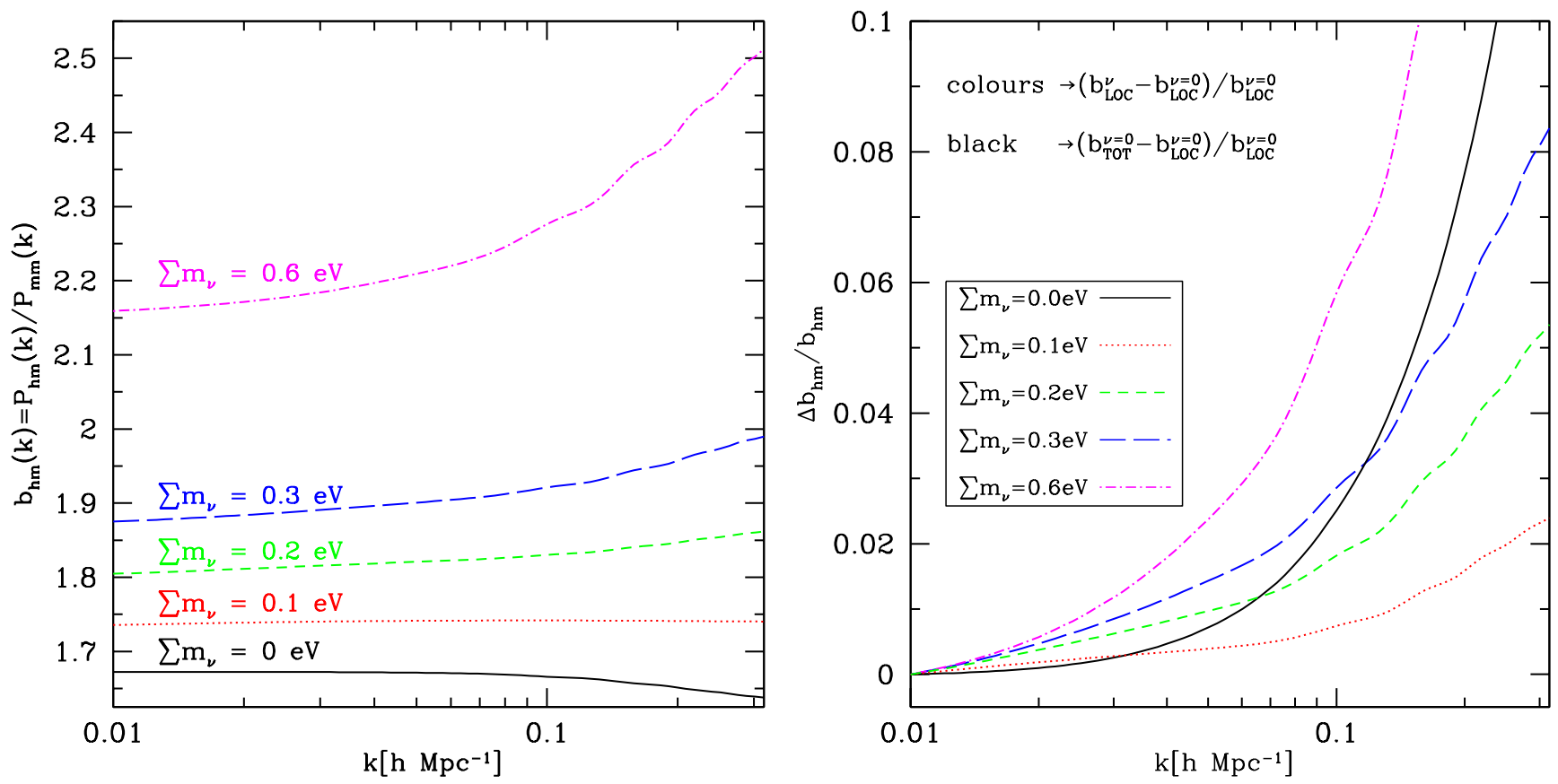

FIG. 2: Halo bias (left) and fractional scale-dependence (right) at $z=0$ as a function of wavenumber for values of $\sum_{i} m_{\nu_{i}}=0$, $0.1,0.2,0.3$ and $0.6 \mathrm{eV}$. In the left panel, only the local bias terms are included in the predictions. In the right panel, the models with non-zero neutrino masses still assume local bias, whereas the solid (black) curve represents the case in which massive neutrinos are absent but all non-local terms are accounted for. The non-local bias contributions induced by gravity and by the peak constraint generate a sharp rise beyond $k \sim 0.1 h \mathrm{Mpc}^{-1}$ substantially steeper than the effect of non-zero neutrino mass.
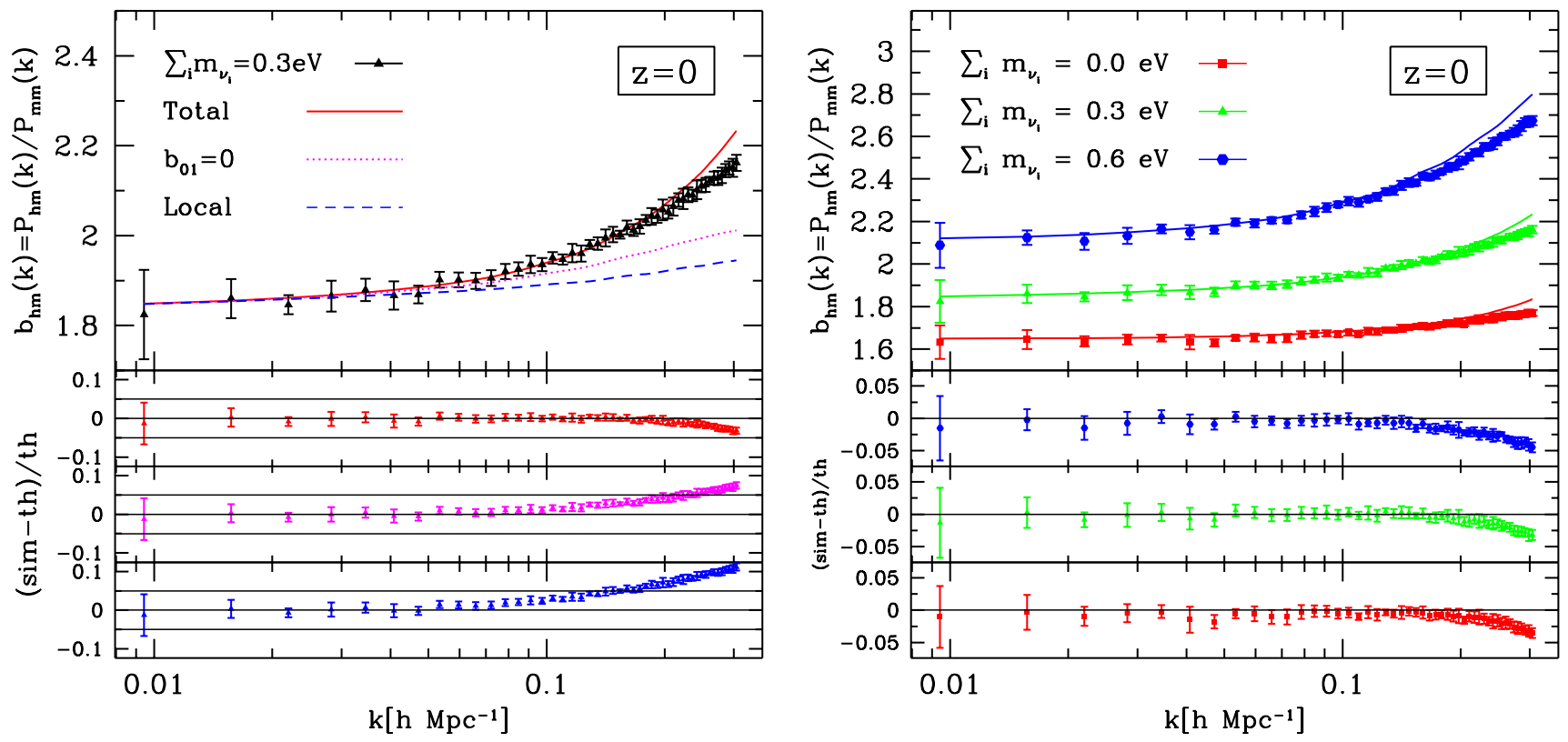

FIG. 3: Halo bias at $z=0$ as a function of wavenumber. Data points are from [22]. In the upper left panel, we show for $\sum_{i} m_{\nu_{i}}=0.3 \mathrm{eV}$ the local bias prediction as the dashed (blue) curve, and our full non-local model as the solid (red) curve. The difference between the solid (red) and the dotted (magenta) curve represents the effect of turning off the contribution $b_{01} k^{2}$ arising from the peak constraint. In the upper right panel, we compare our non-local prediction with the numerical data for $\sum_{i} m_{\nu_{i}}=0,0.3$ and $0.6 \mathrm{eV}$. The lower panels show the fractional deviation between theory and simulations. Note that these predictions have no free parameter. 


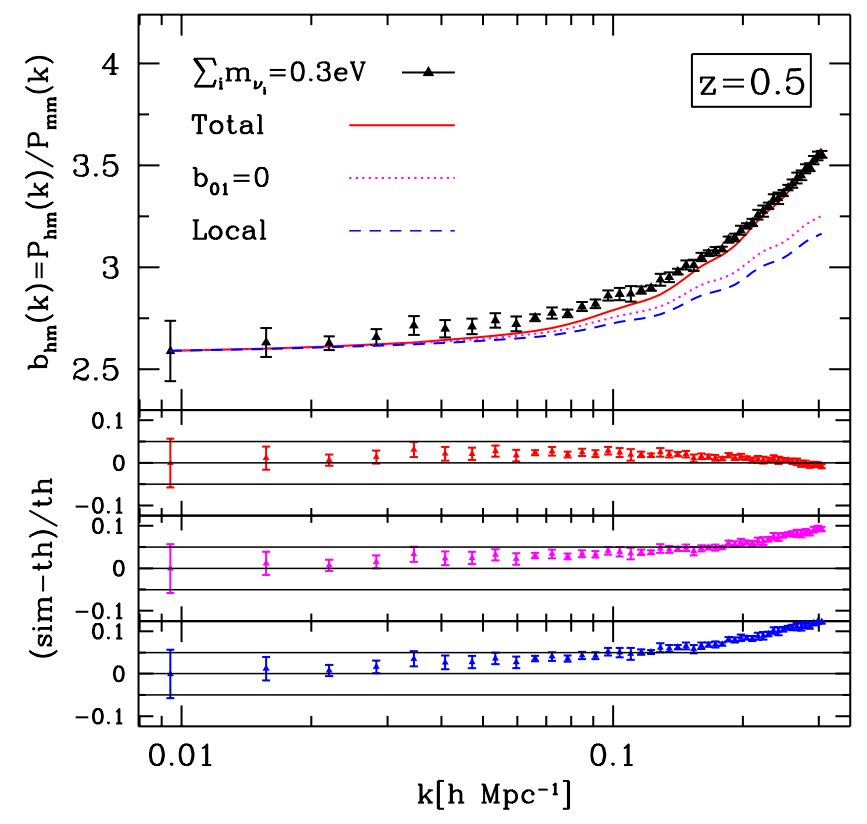

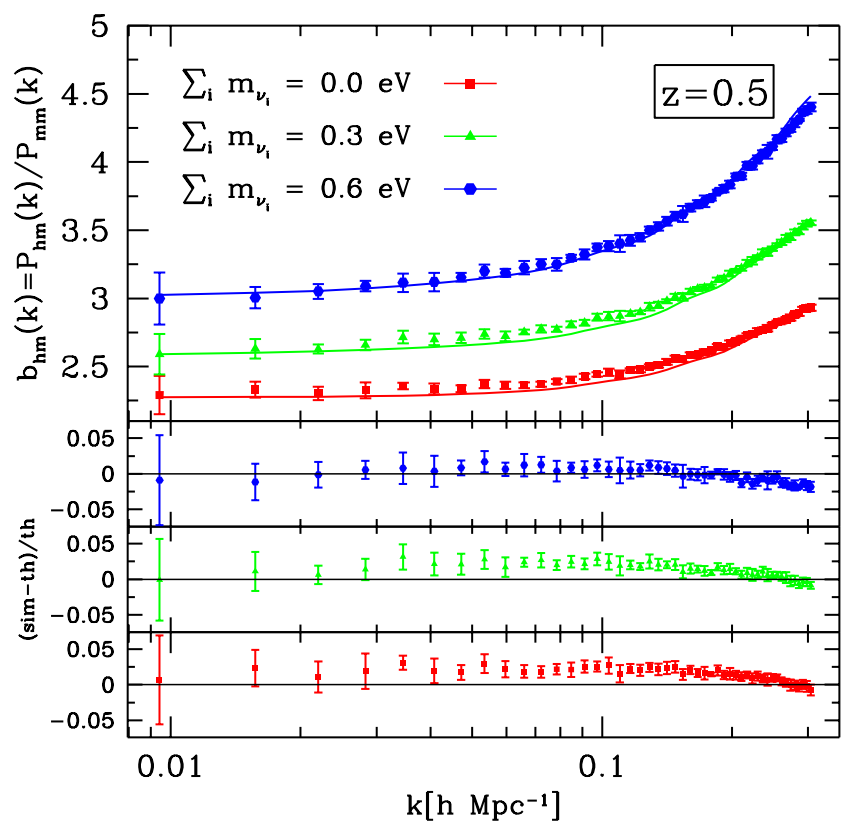

FIG. 4: Same as Fig 3 but at redshift $z=0.5$.

curvature term $\left(b_{01}^{\mathrm{L}}-R_{v}^{2}\right) k^{2}$, which is of positive sign for the mass considered here (see Table 1). It is only partly compensated by the other ones, which are negative or close to zero.

The left panel of Fig. 2 displays the scale-dependence of the halo bias at $z=0$ as a function of neutrino masses. For the sake of illustration, we have assumed the simplest local bias model specified above, in which only $b_{10}=1+b_{10}^{\mathrm{L}}$ and $b_{20}=b_{20}^{\mathrm{L}}+8 / 21 b_{10}^{\mathrm{L}}$ are different from zero. In the right panel of Fig 2 , we show the scale-dependence relative to the $\sum m_{\nu}=0$ case. This should be compared to the solid (black) curve, which represents the scale-dependence obtained when massive neutrinos are absent but all the non-local terms are included. These terms generate a sharp rise beyond $k \sim 0.1 \mathrm{~h} \mathrm{Mpc}^{-1}$ which is much steeper than the effect of varying the sum of neutrino masses. Therefore, a measurement of the scale-dependence of bias over a range of wavenumbers should help disentangling the contribution of massive neutrinos from that induced by the non-local terms.

In Figs 3 and 4 , we compare our predictions for the halo bias at $z=0$ and 0.5 with the N-body measurements of [22]. In both figures, the left panel displays the case of a neutrino sum of $0.3 \mathrm{eV}$. The dashed (blue) curve is the prediction in the simplest local bias model, which falls short of explaining the rise on scales $k \gtrsim 0.1 \mathrm{~h} \mathrm{Mpc}{ }^{-1}$. Our full non-local prediction shown as the solid (red) curve agrees with the numerical data within $3 \%$ for $k \lesssim 0.3 h \mathrm{Mpc}^{-1}$. Most of the difference with the local bias prediction arises from including the peak constraint through the contribution $b_{01} k^{2}$, which is the difference between the solid (red) and dotted (magenta) curve. The right panel compares our full non-local model with the numerical results for $\sum_{i} m_{\nu_{i}}=0,0.3$ and $0.6 \mathrm{eV}$. In all cases the agreement is at the $\sim 3 \%$ level down to $k=0.3 \mathrm{~h} \mathrm{Mpc}^{-1}$. We emphasize again that our theoretical predictions have no free parameter: all the bias factors are consistently determined from the ESP halo mass function and from the relations Eq.(II.54). However, the fact that the magnitude of the $k^{2}$ correction is consistent with $b_{01}^{\mathrm{L}}-R_{v}^{2}$ may be coincidental as we expect similar contributions from higher-order Lagrangian bias. Since we do not have as yet Eulerian expressions for these additional bias contributions, we defer a thorough discussion of this issue to future work.

\section{CONCLUSION}

In light of the expected accuracy of upcoming galaxy surveys, it is of great importance to characterize as well as possible the bias between the matter distribution and the luminous tracers. The relation between the halo overdensity and the underlying DM fluctuations is not local owing to the nonlinear gravitational evolution and to the nature of the initial overdensities which halos collapse form. In the first part of this paper, we have taken another step towards this goal by computing the non-local terms induced by gravity at third-order in perturbation theory. We have computed the coefficients of the non-local operators by expanding the halo density contrast in terms of the DM quantities. 
We have generalized our expressions to include a non-local, linear term induced by a peak constraint in the initial conditions. In the second part of the paper, we have applied our results to model the scale-dependence of bias that arises in cosmologies with non-zero neutrino masses. For the range of halo mass considered here $\left(M \sim 10^{13} h^{-1} M_{\odot}\right)$, the various non-local bias terms conspire to create a steep rise beyond $k \sim 0.1 h \mathrm{Mpc}^{-1}$ which is quite distinct from the gradual scale-dependence generated by the massive neutrinos. We have shown that the inclusion of the non-local bias terms, especially the linear $k^{2}$ correction induced by e.g. a peak constraint in the initial conditions, is crucial for reproducing N-body data if the Eulerian bias factors satisfy the relations Eq.(II.54). Using the ESP halo mass function, we have been able to fit the N-body measurement of [22] to within $\sim 3 \%$ up to $k \sim 0.3 h \mathrm{Mpc}^{-1}$ without $^{2}$ any free parameter. One could envisage further improvements to our computation, e.g. the inclusion of additional Lagrangian bias parameters, to extend the agreement to higher wavenumbers. Clearly however, a more detailed comparison including e.g. several halo mass bins, bispectrum measurements is in order to test the validity of this approach.

On a final note, we became aware of a similar work by S. Saito et al. [48] when completing this work. Our findings agree with theirs wherever there is overlap (basically Eq. (II.43)). In contrast to our approach, Ref. [48 does not include $k^{2}$-bias. However, they treat the amplitude of $b_{\mathrm{NL}}$ as free parameter and include additional constraints from the bispectrum, so there is no real contradiction. Furthermore, their best-fit values of $b_{\mathrm{NL}}$ are somewhat larger than the expectation $(32 / 315) b_{10}^{\mathrm{L}}$ for $b_{10} \lesssim 2$, a discrepancy which could be partly resolved with the inclusion of $k^{2}$ bias.

\section{Acknowledgments}

It is a pleasure to thank M. Viel and F. Villaescusa-Navarro for kindly making their N-body data available to us, Emiliano Sefusatti for helpful discussions, and S. Saito for correspondence about Ref. 48. M.B. would also like to thank Enea Di Dio and Francesco Montanari for useful advice about the CLASS code. The research of A.K. was implemented under the Aristeia Action of the Operational Programme Education and Lifelong Learning and is co-funded by the European Social Fund (ESF) and National Resources. A.K. is also partially supported by European Unions Seventh Framework Programme (FP7/2007-2013) under REA grant agreement n. 329083. A.R. is supported by the Swiss National Science Foundation (SNSF), project "The non-Gaussian Universe" (project number: 200021140236). M.B. and V.D. acknowledge support by the Swiss National Science Foundation.

[1] J. N. Fry and E. Gaztanaga, Astrophys. J. 413, 447 (1993) astro-ph/9302009.

[2] P. J. E. Peebles, Princeton University Press (1980).

[3] J. N. Fry, Astrophys. J. 279, 499 (1984).

[4] M. H. Goroff, B. Grinstein, S. J. Rey and M. B. Wise, Astrophys. J. 311, 6 (1986).

[5] F. R. Bouchet, R. Juszkiewicz, S. Colombi and R. Pellat, Astrophys. J. 394, L5 (1992).

[6] P. Catelan, F. Lucchin, S. Matarrese, C. Porciani, Mon. Not. R. Astron. Soc. 297, 692 (1998).

[7] P. Catelan, C. Porciani and M. Kamionkowski, Mon. Not. Roy. Astron. Soc. 318, 39 (2000) astro-ph/0005544.

[8] P. McDonald and A. Roy, JCAP 0908, 020 (2009) arXiv:0902.0991 [astro-ph.CO]].

[9] V. Desjacques, M. Crocce, R. Scoccimarro, R.K. Sheth, Phys. Rev. D 82, 103529 (2010) arXiv:1009.3449 [astro-ph.CO]].

[10] T. Matsubara, Phys. Rev. D 83, 083518 (2011) arXiv:1102.4619 [astro-ph.CO]].

[11] K. C. Chan, R. Scoccimarro and R. K. Sheth, Phys. Rev. D 85, 083509 (2012) arXiv:1201.3614 [astro-ph.CO]].

[12] T. Baldauf, U. Seljak, V. Desjacques and P. McDonald, Phys. Rev. D 86, 083540 (2012) arXiv:1201.4827 [astro-ph.CO]].

[13] A. Kehagias, J. .Noreña, H. Perrier and A. Riotto, arXiv:1311.0786 [astro-ph.CO].

[14] S. Saito, M. Takada, A. Taruya, Phys. Rev. D 83, 043529 (2011), arXiv:1006.4845 [astro-ph.CO].

[15] B. Audren, J. Lesgourgues, S. Bird, M. G. Haehnelt, M. Viel, JCAP 01, 026 (2013),

[16] G.-B. Zhao, S. Saito, W. J. Percival, A. J. Ross, F. Montesano, M. Viel, D. P. Schneider, M. Manera, J. Miralda-Escudé, N. Palanque-Delabrouille, N. P. Ross, L. Samushia, A.G. Sánchez, M. E. C. Swanson, D. Thomas, R. Tojeiro, C. Yèche, D. G. York, Mon. Not. Roy. Astron. Soc. 436, 2038 (2013) arXiv:1211.3741 [astro-ph.CO]].

[17] A. Font-Ribera, P. M. McDonald, N. Mostek, B. A. Reid, H.-J. Seo, A. Slosar, arXiv:1308.4164 [astro-ph.CO]].

[18] F. Beutler, S. Saito, H.-J. Seo, J. Brinkmann, K. S. Dawson, D. J. Eisenstein et al. arXiv:1312.4611 [astro-ph.CO]].

[19] M. LoVerde, arXiv:1405.4858 [astro-ph.CO].

[20] J. Lesgourgues and S. Pastor, Phys. Rept. 429, 307 (2006) astro-ph/0603494.

[21] J. Lesgourgues and S. Pastor, Adv. High Energy Phys. 2012, 608515 (2012) arXiv:1212.6154 [hep-ph]].

[22] F. Villaescusa-Navarro, F. Marulli, M. Viel, E. Branchini, E. Castorina, E. Sefusatti and S. Saito, arXiv:1311.0866 [astroph.CO].

[23] E. Castorina, E. Sefusatti, R. K. Sheth, F. Villaescusa-Navarro and M. Viel, JCAP 1402 (2014) 049 arXiv:1311.1212 [astro-ph.CO]]. 
[24] M. Costanzi, F. Villaescusa-Navarro, M. Viel, J. -Q. Xia, S. Borgani, E. Castorina and E. Sefusatti, JCAP 1312 (2013) 012 arXiv:1311.1514 [astro-ph.CO]].

[25] M. LoVerde, arXiv:1405.4855 [astro-ph.CO].

[26] A. Elia, S. Kulkarni, C. Porciani, M. Pietroni and S. Matarrese, Mon. Not. Roy. Astron. Soc. 416, 1703 (2011) arXiv:1012.4833 [astro-ph.CO]].

[27] P.J.E. Peebles, The large-scale structure of the universe (Princeton University Press, Princeton, NJ, 1980)

[28] N. Bartolo, S. Matarrese and A. Riotto, JCAP 0510, 010 (2005) astro-ph/0501614; N. Bartolo, S. Matarrese, O. Pantano and A. Riotto, Class. Quant. Grav. 27, 124009 (2010) arXiv:1002.3759 [astro-ph.CO]] and references therein.

[29] V. Desjacques, Phys. Rev. D 78, 103503 (2008) arXiv:0806.0007 [astro-ph]].

[30] V. Desjacques, Phys. Rev. D 87, no. 4, 043505 (2013) arXiv:1211.4128 [astro-ph.CO]].

[31] L. Verde, R. Jimenez, F. Simpson, L. Alvarez-Gaume, A. Heavens and S. Matarrese, arXiv:1404.2241 [astro-ph.CO].

[32] V. Desjacques and R. K. Sheth, Phys. Rev. D 81, 023526 (2010) arXiv:0909.4544 [astro-ph.CO]].

[33] T. Baldauf, V. Desjacques and U. Seljak, arXiv:1405.5885 [astro-ph.CO].

[34] S. Saito, M. Takada, A. Taruya, Phys. Rev. D 80, 083528 (2009) arXiv:0907.2922 [astro-ph.CO]].

[35] Y. Y. Y. Wong, JCAP 0810, 035 (2008) arXiv:0809.0693 [astro-ph]].

[36] K. Ichiki, M. Takada, Phys. Rev. D 85, 063521 (2012) arXiv:1108.4688 [astro-ph.CO]].

[37] M. Shoji, E. Komatsu, Phys. Rev. D 81, 123516 (2010)

[38] P. McDonald, Phys. Rev. D 74, 103512 (2006) [Erratum-ibid. D 74, 129901 (2006)] astro-ph/0609413.

[39] D. Blas, J. Lesgourgues and T. Tram, JCAP 1107 (2011) 034 arXiv:1104.2933 [astro-ph.CO]].

[40] J. Lesgourgues, arXiv:1104.2932 [astro-ph.IM].

[41] S. Bird, M. Viel and M. G. Haehnelt, Mon. Not. Roy. Astron. Soc. 420 (2012) 2551 arXiv:1109.4416 [astro-ph.CO]].

[42] A. Paranjape and R. K. Sheth, Mon. Not. Roy. Astron. Soc. 426 (2012) 2789 [arXiv:1206.3506 [astro-ph.CO]].

[43] A. Paranjape, R. K. Sheth and V. Desjacques, Mon. Not. Roy. Astron. Soc. 431 (2013) 1503 arXiv:1210.1483 [astroph.CO]].

[44] A. Paranjape, E. Sefusatti, K. C. Chan, V. Desjacques, P. Monaco and R. K. Sheth, Mon. Not. Roy. Astron. Soc. 436, 449 (2013) arXiv:1305.5830 [astro-ph.CO]].

[45] M. Biagetti, K. C. Chan, V. Desjacques and A. Paranjape, arXiv:1310.1401 [astro-ph.CO].

[46] O. Hahn and A. Paranjape, Mon. Not. Roy. Astron. Soc. 438, 878 (2014) arXiv:1308.4142 [astro-ph.CO]].

[47] The simulations were seeded with a transfer function output from a Boltzmann code at $z=z_{i}$ rather than at $z=0$, where $z_{i}$ is the starting redshift, and subsequently ignore the contribution of radiation to the Hubble rate. Therefore, for the sake of comparison we set $\rho_{\gamma}=0$ in the CLASS code when $0 \leq z \leq z_{i}$. This translates into a few percent increase in the linear growth rate at $z=0$, and mainly has an impact on our prediction of $b_{10}$.

[48] S. Saito, T. Baldauf, Z. Vlah, U. Seljak, T. Okumura, P. M. McDonald, arXiv:1405.1447] [astro-ph.CO].

\section{Appendix A: The halo-mass correlator}

The typical integral we need to calculate is

$$
\mathcal{I}_{i j}(k)=\int \frac{\mathrm{d}^{3} q}{(2 \pi)^{3}} P_{i}(q) P_{j}(|\vec{k}-\vec{q}|) F_{2}(\vec{q}, \vec{k}-\vec{q})
$$

where

$$
F_{2}\left(\vec{q}_{1}, \vec{q}_{2}\right)=\frac{5}{7}+\frac{1}{2} \frac{\vec{q}_{1} \cdot \vec{q}_{2}}{q_{1} q_{2}}\left(\frac{q_{1}}{q_{2}}+\frac{q_{2}}{q_{1}}\right)+\frac{2}{7}\left(\frac{\vec{q}_{1} \cdot \vec{q}_{2}}{q_{1} q_{2}}\right)^{2} .
$$

Furthermore

$$
S\left(\vec{q}_{1}, \vec{q}_{2}\right)=\left(\frac{\vec{q}_{1} \cdot \vec{q}_{2}}{q_{1} q_{2}}\right)^{2}-\frac{1}{3}
$$

We now define the following variables

$$
\begin{aligned}
& \mu=\frac{\vec{k} \cdot \vec{q}}{k q} \\
& r=\frac{q}{k}
\end{aligned}
$$

such that 


$$
\begin{aligned}
\int \frac{\mathrm{d}^{3} q}{(2 \pi)^{3}} & =\frac{k^{3}}{(2 \pi)^{2}} \int_{0}^{\infty} r^{2} \mathrm{~d} r \int_{-1}^{1} \mathrm{~d} \mu, \\
\eta k & \equiv|\vec{k}-\vec{q}|=\sqrt{q^{2}+k^{2}-2 \vec{k} \cdot \vec{q}}=k \sqrt{r^{2}+1-2 r \mu}, \\
F_{2}(\vec{q}, \vec{k}-\vec{q}) & =\frac{5}{7}+\frac{1}{2}\left[(\vec{k} \cdot \vec{q})-q^{2}\right]\left(\frac{1}{q^{2}}+\frac{1}{k^{2} \eta^{2}}\right)+\frac{2}{7} \frac{\left[(\vec{k} \cdot \vec{q})-q^{2}\right]^{2}}{q^{2} k^{2} \eta^{2}} \\
& =\frac{5}{7}+\frac{1}{2}\left(\mu r-r^{2}\right) \frac{2 r^{2}+1-2 r \mu}{r^{2} \eta^{2}}+\frac{2}{7} \frac{\left(\mu r-r^{2}\right)^{2}}{r^{2} \eta^{2}} \\
& =\frac{3 r+7 \mu-10 \mu^{2} r}{14 r\left(1+r^{2}-2 \mu r\right)} \\
S(\vec{q}, \vec{k}-\vec{q}) & =\frac{\left(\mu r-r^{2}\right)^{2}}{r^{2} \eta^{2}}-\frac{1}{3} .
\end{aligned}
$$

We finally obtain

$$
\mathcal{I}_{i j}(k)=\frac{k^{3}}{56 \pi^{2}} \int_{0}^{\infty} \mathrm{d} r \int_{-1}^{1} \mathrm{~d} \mu P_{i}(k r) P_{j}\left(k \sqrt{r^{2}+1-2 r \mu}\right) \frac{r\left(3 r+7 \mu-10 \mu^{2} r\right)}{\left(1+r^{2}-2 \mu r\right)}
$$

Notice that, in order to avoid the IR divergence when $|\vec{k}-\vec{q}|$ goes to zero, by exploring the symmetry of the integral, we can rewrite it as

$$
\begin{aligned}
\mathcal{I}_{i j}(k) & =\int \frac{\mathrm{d}^{3} q}{(2 \pi)^{3}} P_{i}(q) P_{j}(|\vec{k}-\vec{q}|) F_{2}(\vec{q}, \vec{k}-\vec{q}) \Theta(|\vec{k}-\vec{q}|-q)+(i \leftrightarrow j) \\
& =\frac{k^{3}}{56 \pi^{2}} \int_{0}^{\infty} \mathrm{d} r \int_{-1}^{1} \mathrm{~d} \mu P_{i}(k r) P_{j}\left(k \sqrt{r^{2}+1-2 r \mu}\right) \frac{r\left(3 r+7 \mu-10 \mu^{2} r\right)}{\left(1+r^{2}-2 \mu r\right)} \Theta(1-2 r \mu)+(i \leftrightarrow j)
\end{aligned}
$$

where $\Theta(x)$ is the Heaviside step function. Similarly

$$
\begin{aligned}
\int \frac{\mathrm{d}^{3} q}{(2 \pi)^{3}} P_{i}(q) P_{j}(|\vec{k}-\vec{q}|) F_{2}(\vec{q}, \vec{k}-\vec{q}) S(\vec{q}, \vec{k}-\vec{q}) & =\frac{k^{3}}{168 \pi^{2}} \int_{0}^{\infty} \mathrm{d} r \int_{-1}^{1} \mathrm{~d} \mu P_{i}(k r) P_{j}\left(k \sqrt{r^{2}+1-2 r \mu}\right) \\
& \times \frac{\left(3 r+7 \mu-10 \mu^{2} r\right)\left(1-3 \mu^{2}+4 \mu r-2 r^{2}\right)}{r\left(1+r^{2}-2 \mu r\right)^{2}} .
\end{aligned}
$$

